\title{
Synthesis and Structures of Novel Luminescent Bent Acenedithiophenes
}

Agostino Pietrangelo, Mark J. MacLachlan ${ }^{*}$, Michael O. Wolf ${ }^{*}$, and Brian O. Patrick

Department of Chemistry, University of British Columbia, 2036 Main Mall, Vancouver, B.C. V6T $1 Z 1$

mmaclach@chem.ubc.ca

mwolf@chem.ubc.ca

Supporting Information 
Table of Contents

$\begin{array}{ll}\text { Experimental Methods and Characterization } & \mathrm{S}-2\end{array}$

Figure 1. ${ }^{1} \mathrm{H}$ NMR spectrum of 3a. $\quad$ S-7

Figure 2. ${ }^{13} \mathrm{C}$ NMR spectrum of 3a. $\quad$ S-7

Figure 3. ${ }^{1} \mathrm{H}$ NMR spectrum of $\mathbf{3 b}$.

Figure 4. ${ }^{13} \mathrm{C}$ NMR spectrum of $3 \mathbf{b}$. S-8

Figure 5. ${ }^{1} \mathrm{H}$ NMR spectrum of 3c. $\quad$ S-9

Figure 6. ${ }^{13} \mathrm{C}$ NMR spectrum of $3 c . \quad$ S-9

Figure 7. ${ }^{1} \mathrm{H}$ NMR spectrum of 3d. $\quad$ S-10

Figure 8. ${ }^{13} \mathrm{C}$ NMR spectrum of $3 \mathbf{d}$. $\quad$ S-10

Figure 9. ${ }^{1} \mathrm{H}$ NMR spectrum of 4a. $\quad$ S-11

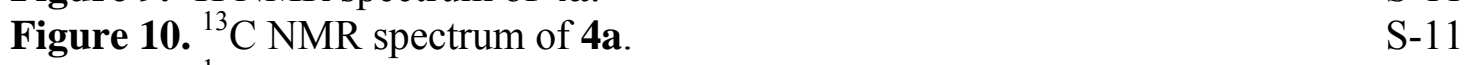

Figure 11. ${ }^{1} \mathrm{H}$ NMR spectrum of $\mathbf{4 b}$. $\quad$ S-12

Figure 12. ${ }^{13} \mathrm{C}$ NMR spectrum of $\mathbf{4 b}$.

Figure 13. ${ }^{1} \mathrm{H}$ NMR spectrum of 4c. $\quad$ S-13

Figure 14. ${ }^{13} \mathrm{C}$ NMR spectrum of 4c. $\quad$ S-13

Figure 15. ${ }^{1} \mathrm{H}$ NMR spectrum of 4d. $\quad$ S-14

Figure 16. ${ }^{13} \mathrm{C}$ NMR spectrum of 4d. $\quad$ S-14

Figure 17. ${ }^{1} \mathrm{H}$ NMR spectrum of 5a. $\quad$ S-15

Figure 18. ${ }^{13} \mathrm{C}$ NMR spectrum of 5a. $\quad \mathrm{S}-15$

Figure 19. ${ }^{1} \mathrm{H}$ NMR spectrum of $5 \mathbf{b}$. $\quad$ S-16

Figure 20. ${ }^{13} \mathrm{C}$ NMR spectrum of $5 \mathbf{b}$. $\quad$ S-16

$\begin{array}{ll}\text { Figure 21. }{ }^{1} \mathrm{H} \text { NMR spectrum of 5c. } & \mathrm{S}-17\end{array}$

Figure 22. ${ }^{13} \mathrm{C}$ NMR spectrum of 5c. $\quad$ S-17

Figure 23. ${ }^{1} \mathrm{H}$ NMR spectrum of 5d. $\quad$ S-18

Figure 24. ${ }^{13} \mathrm{C}$ NMR spectrum of 5d. $\quad \mathrm{S}-18$

Figure 25. UV/vis absorption spectrum of 5a. $\quad$ S-19

Figure 26. UV/vis absorption spectrum of $\mathbf{5 b}$. $\quad$ S-19

Figure 27. UV/vis absorption spectrum of 5c. $\quad$ S-20

Figure 28. UV/vis absorption spectrum of 5d. S-20

Figure 29. Fluorescence spectrum of 5a. $\quad \mathrm{S}-21$

Figure 30. Fluorescence spectrum of 5b. $\quad$ S-21

Figure 31. Fluorescence spectrum of 5c. $\quad$ S-22

Figure 32. Fluorescence spectrum of 5d. S-22

\section{Experimental Section}

Materials and Equipment. Chromium trioxide, 2-(tributylstannyl)thiophene, anhydrous dimethylformamide (DMF), $p$-xylene, and methyltriphenylphosphonium bromide were purchased from Aldrich. Tetrakis(triphenylphosphine) palladium (0) was purchased from Strem. Tetrahydrofuran (THF) was distilled from sodium and benzophenone under nitrogen gas. Deuterated solvents were purchased from Cambridge Isotope Laboratories, Inc. All purchased chemicals were used without further purification. 2,5Dibromobenzene $^{1}, \quad$ 2,5-dibromoterephthalaldehyde ${ }^{2}, \quad$ 5-methyl-2- 
(tributylstannyl)thiophene ${ }^{3}$, 5-hexyl-2-(tributylstannyl)thiophene ${ }^{4}$, and 5-dodecyl-2(tributylstannyl)thiophene ${ }^{5}$ were prepared using literature methods. All reactions were carried out under a nitrogen atmosphere unless otherwise noted. All photochemical reactions were carried out in a Rayonet Photoreactor (Southern New England Ultraviolet Company, Branford, CT) equipped with 16 RPR-3000 $\left(300 \mathrm{~nm}, 21 \mathrm{~W}\right.$ ) lamps. ${ }^{1} \mathrm{H}$ NMR spectra were recorded on either a Bruker AV-300 or AV-400 spectrometer and calibrated to the residual protonated solvent at $\delta 5.32$ for deuterated dichloromethane $\left(\mathrm{CD}_{2} \mathrm{Cl}_{2}\right)$ and $\delta 7.24$ for deuterated chloroform $\mathrm{CDCl}_{3}$. All ${ }^{13} \mathrm{C}$ NMR experiments were carried out in $\mathrm{CD}_{2} \mathrm{Cl}_{2}$ and calibrated at $\delta$ 54.00. Solution absorption spectra were obtained in dichloromethane (DCM) on a Varian Cary 5000 UV-vis-near-IR spectrophotometer using a $1 \mathrm{~cm}$ quartz cuvette. IR spectra were obtained as $\mathrm{KBr}$ discs on a Bomem MB-100 FTIR spectrometer. Fluorescence spectra were obtained in DCM on a Photon Technology International QuantaMaster fluorimeter using a $1 \mathrm{~cm}$ quartz cuvette. Quantum Yields were referenced to a solution of anthracene in EtOH. $\left(\Phi_{\mathrm{f}}=0.3\right) .{ }^{6}$ Melting points were obtained on a Fisher-Johns melting point apparatus. Electron ionization (EI) mass spectra were obtained at the UBC Mass Spectrometry facility. Elemental analyses were obtained at the UBC Microanalytical facility. Single crystal x-ray diffraction measurements were made on a Bruker X8 APEX diffractometer with graphite monochromated Mo-Ka radiation. Powder X-ray diffraction measurements were made on a Bruker D8 Advance diffractometer with graphite monochromated $\mathrm{Cu}-\mathrm{K} \alpha$ radiation. Films of $\mathbf{5 a}$ and $\mathbf{5 d}$ were prepared by vacuum evaporation onto glass substrates at $150^{\circ} \mathrm{C}$ and $200^{\circ} \mathrm{C}$ respectively, at a pressure of $\sim 0.1$ to 1 Torr.

Synthesis of 2,5-di(2-thienyl)terephthalaldehyde (3a). A $100 \mathrm{~mL}$ round bottom Schlenk flask was charged with 2,5-dibromoterephthalaldehyde $2(2.00 \mathrm{~g}, 6.85 \mathrm{mmol})$, tetrakis(triphenylphosphine)palladium $(0) \quad(800 \mathrm{mg}, \quad 0.692 \mathrm{mmol}), \quad 2$-(tributylstannyl)thiophene $(7.68 \mathrm{~g}, 20.6 \mathrm{mmol})$ and anhydrous DMF $(60 \mathrm{~mL})$ and heated to $80^{\circ} \mathrm{C}$ with stirring for $72 \mathrm{~h}$. The reaction mixture was then cooled to room temperature, diluted with $\mathrm{Et}_{2} \mathrm{O}$, and filtered through Celite. The organic phase was washed with brine and water and dried over $\mathrm{MgSO}_{4}$. After filtration, solvent was removed to yield the crude product that was then chromatographed on silica using a hexane $/ \mathrm{CH}_{2} \mathrm{Cl}_{2}(2: 1)$ solvent mixture as the eluent $\left(\mathrm{R}_{\mathrm{f}}\right.$ : 0.29$)$. The desired product was a crystalline yellow solid $(1.35 \mathrm{~g}$, yield $66 \%) .{ }^{1} \mathrm{H}$ NMR $\left(\mathrm{CD}_{2} \mathrm{Cl}_{2}, 300 \mathrm{MHz}\right) \delta 10.25(2 \mathrm{H}, \mathrm{s}, \mathrm{CHO}), 8.12(2 \mathrm{H}, \mathrm{s}$, aromatic $\mathrm{CH})$, $7.57\left(2 \mathrm{H}, \mathrm{dd},{ }^{3} \mathrm{~J}_{\mathrm{HH}}=4.97 \mathrm{~Hz},{ }^{3} \mathrm{~J}_{\mathrm{HH}}=1.28 \mathrm{~Hz}\right.$, aromatic $\left.\mathrm{CH}\right), 7.19(4 \mathrm{H}, \mathrm{m}$, aromatic $\mathrm{CH})$. ${ }^{13} \mathrm{C}$ NMR $\left(\mathrm{CD}_{2} \mathrm{Cl}_{2}, 100 \mathrm{MHz}\right) \delta 191.6,138.0,137.5,137.4,131.3,130.7,128.8,128.6$. EI-MS: $m / z=298\left(\mathrm{M}^{+}, 100 \%\right)$. UV-vis $\left(\mathrm{CH}_{2} \mathrm{Cl}_{2}\right): \lambda_{\max }(\varepsilon)=374(4600), 269(29600) \mathrm{nm}$ $\left(\mathrm{L} \mathrm{mol}^{-1} \mathrm{~cm}^{-1}\right)$. IR $(\mathrm{KBr}): v=1684,1529,1431,1384,1327,1279,1144,846,818,721$ $\mathrm{cm}^{-1}$. Mp. $160-162^{\circ} \mathrm{C}$. Anal. Calcd for $\mathrm{C}_{16} \mathrm{H}_{10} \mathrm{O}_{2} \mathrm{~S}_{2}$ : C, 64.41; H, 3.38. Found: C, 64.61, $\mathrm{H}, 3.42$.

Synthesis of 2,5-di(5-methyl-2-thienyl)terephthalaldehyde (3b). The same procedure was used as for preparation of $3 \mathbf{a}$. $\mathrm{R}_{\mathrm{f}}=0.38$ (hexane $/ \mathrm{CH}_{2} \mathrm{Cl}_{2}(2: 1)$ ). The product was isolated as an orange crystalline solid (yield $72 \%) .{ }^{1} \mathrm{H}$ NMR $\left(\mathrm{CD}_{2} \mathrm{Cl}_{2}, 400 \mathrm{MHz}\right) \delta 10.25$ $(2 \mathrm{H}, \mathrm{s}, \mathrm{CHO}), 8.05(2 \mathrm{H}, \mathrm{s}$, aromatic $\mathrm{CH}), 6.94\left(2 \mathrm{H}, \mathrm{d},{ }^{3} J_{\mathrm{HH}}=3.46 \mathrm{~Hz}\right.$, aromatic $\left.\mathrm{CH}\right)$, $6.86(2 \mathrm{H}, \mathrm{m}$, aromatic $\mathrm{CH}), 2.57\left(6 \mathrm{H}, \mathrm{s}, \mathrm{CH}_{3}\right) .{ }^{13} \mathrm{C} \mathrm{NMR}\left(\mathrm{CD}_{2} \mathrm{Cl}_{2}, 100 \mathrm{MHz}\right) \delta 191.8$, 
144.0, 137.4, 137.2, 135.6, 131.0, 130.7, 127.0, 15.7. EI-MS: $\mathrm{m} / \mathrm{z}=326.1\left(\mathrm{M}^{+}, 100 \%\right)$. UV-vis $\left(\mathrm{CH}_{2} \mathrm{Cl}_{2}\right): \lambda_{\max }(\varepsilon)=401(5600), 308(17800), 271(24700) \mathrm{nm}\left(\mathrm{L} \mathrm{mol}^{-1} \mathrm{~cm}^{-1}\right)$. IR $(\mathrm{KBr}): v=1684,1560,1460,1399,1278,1146,912,824,796,720 \mathrm{~cm}^{-1}$. Mp. 164-166 ${ }^{\circ} \mathrm{C}$. Anal. Calcd for $\mathrm{C}_{18} \mathrm{H}_{14} \mathrm{O}_{2} \mathrm{~S}_{2}$ : C, 66.23; H, 4.32. Found: C, 66.64; H, 4.32

Synthesis of 2,5-di(5-hexyl-2-thienyl)terephthalaldehyde (3c). The same procedure was used as for preparation of $3 \mathbf{a}$. $\mathrm{R}_{\mathrm{f}}=0.58$ (hexane $/ \mathrm{CH}_{2} \mathrm{Cl}_{2}(2: 1)$ ). The product was isolated as a yellow crystalline solid (yield $87 \%) .{ }^{1} \mathrm{H}$ NMR $\left(\mathrm{CD}_{2} \mathrm{Cl}_{2}, 400 \mathrm{MHz}\right) \delta 10.26$ $(2 \mathrm{H}, \mathrm{s}, \mathrm{CHO}), 8.06(2 \mathrm{H}, \mathrm{s}$, aromatic $\mathrm{CH}), 6.95\left(2 \mathrm{H}, \mathrm{d},{ }^{3} J_{\mathrm{HH}}=3.50 \mathrm{~Hz}\right.$, aromatic $\left.\mathrm{CH}\right)$, $6.87\left(2 \mathrm{H}, \mathrm{d},{ }^{3} J_{\mathrm{HH}}=3.50 \mathrm{~Hz}\right.$, aromatic $\left.\mathrm{CH}\right), 2.88\left(4 \mathrm{H}, \mathrm{t},{ }^{3} J_{\mathrm{HH}}=7.60 \mathrm{~Hz}, \mathrm{CH}_{2}\right), 1.69-1.77$ $\left(4 \mathrm{H}, \mathrm{m}, \mathrm{CH}_{2}\right), 1.45-1.32\left(12 \mathrm{H}, \mathrm{m}, \mathrm{CH}_{2}\right), 0.91\left(6 \mathrm{H}, \mathrm{t},{ }^{3} \mathrm{~J}_{\mathrm{HH}}=6.99 \mathrm{~Hz}, \mathrm{CH}_{3}\right) \cdot{ }^{13} \mathrm{C} \mathrm{NMR}$ $\left(\mathrm{CD}_{2} \mathrm{Cl}_{2}, 100 \mathrm{MHz}\right) \delta 191.9,150.2,137.4,137.2,135.4,131.0,130.5,125.8,32.2,32.1$, 30.7, 29.4, 23.2, 14.4. EI-MS: $m / z=466\left(\mathrm{M}^{+}, 100 \%\right)$. UV-vis $\left(\mathrm{CH}_{2} \mathrm{Cl}_{2}\right): \lambda_{\max }(\varepsilon)=403$ (5500), 311 (18800), $272(23500) \mathrm{nm}\left(\mathrm{L} \mathrm{mol}^{-1} \mathrm{~cm}^{-1}\right)$. IR (KBr): $v=2953,2924,2853$, $1683,1490,1465,1465,1395,1382,1282,1256,1151,1053,905,818 \mathrm{~cm}^{-1}$. Mp. $90^{\circ} \mathrm{C}$. Anal. Calcd for $\mathrm{C}_{28} \mathrm{H}_{34} \mathrm{O}_{2} \mathrm{~S}_{2}$ : C, 72.06; H, 7.34. Found: C, 72.38; H, 7.42.

Synthesis of 2,5-bis(5-dodecyl-2-thienyl)terephthalaldehyde (3d). The same procedure was used as for preparation of 3a. $\mathrm{R}_{\mathrm{f}}=0.69$ (hexane $/ \mathrm{CH}_{2} \mathrm{Cl}_{2}(2: 1)$ ). The product was recrystallized in hexanes and isolated as a yellow crystalline solid (yield 46\%). ${ }^{1} \mathrm{H}$ NMR $\left(\mathrm{CD}_{2} \mathrm{Cl}_{2}, 400 \mathrm{MHz}\right) \delta 10.26(2 \mathrm{H}, \mathrm{s}, \mathrm{CHO}), 8.06(2 \mathrm{H}, \mathrm{s}$, aromatic $\mathrm{CH}), 6.95\left(2 \mathrm{H}, \mathrm{d},{ }^{3} J_{\mathrm{HH}}=\right.$ $3.48 \mathrm{~Hz}$, aromatic $\mathrm{CH}), 6.87\left(2 \mathrm{H}, \mathrm{d},{ }^{3} \mathrm{~J}_{\mathrm{HH}}=3.45 \mathrm{~Hz}\right.$, aromatic $\left.\mathrm{CH}\right), 2.88\left(4 \mathrm{H}, \mathrm{t},{ }^{3} \mathrm{~J}_{\mathrm{HH}}=\right.$ $\left.7.54 \mathrm{~Hz}, \mathrm{CH}_{2}\right), 1.77-1.69\left(4 \mathrm{H}, \mathrm{m}, \mathrm{CH}_{2}\right), 1.41-1.28\left(36 \mathrm{H}, \mathrm{m}, \mathrm{CH}_{2}\right), 0.88\left(6 \mathrm{H}, \mathrm{t},{ }^{3} \mathrm{~J}_{\mathrm{HH}}=\right.$ $\left.6.53 \mathrm{~Hz}, \mathrm{CH}_{3}\right) .{ }^{13} \mathrm{C}$ NMR $\left(\mathrm{CD}_{2} \mathrm{Cl}_{2}, 100 \mathrm{MHz}\right) \delta 191.88,150.23,137.45,137.18,135.36$, $130.96,130.50,125.78,32.50,32.27,30.74,30.24,30.22,30.13,29.93,29.90,29.68$, 23.27, 14.45. EI-MS: $m / z=634\left(\mathrm{M}^{+}, 100 \%\right)$. UV-vis $\left(\mathrm{CH}_{2} \mathrm{Cl}_{2}\right): \lambda_{\max }(\varepsilon)=403(5400)$, 311 (18000), $272(22400) \mathrm{nm}\left(\mathrm{L} \mathrm{mol}^{-1} \mathrm{~cm}^{-1}\right)$. IR (KBr): v 2918, 2850, 1684, 1491, 1471, 1382, 1285, 1154, 1096, 905, $817 \mathrm{~cm}^{-1}$. Mp. 97-98 ${ }^{\circ} \mathrm{C}$. Anal. Calcd for $\mathrm{C}_{40} \mathrm{H}_{58} \mathrm{O}_{2} \mathrm{~S}_{2}: \mathrm{C}$, 75.66; H, 9.21. Found: C, 75.70, H, 9.23.

Synthesis of 2,5-bis(2-thienyl)-1,4-divinylbenzene (4a). A suspension of methyltriphenylphosphonium bromide $(1.15 \mathrm{~g}, 3.22 \mathrm{mmol})$ and $60 \mathrm{~mL}$ of anhydrous THF in a $250 \mathrm{~mL}$ round bottom Schlenk flask was cooled to $0^{\circ} \mathrm{C}$ and left to stir for $30 \mathrm{~min}$. With stirring, $1.84 \mathrm{~mL}$ of $n-\mathrm{BuLi}(1.6 \mathrm{M}$ in hexanes) was added dropwise. After an additional $30 \mathrm{~min}$ of stirring, a solution of 2,5-di(2-thienyl)terephthalaldehyde 3a (400mg, $1.34 \mathrm{mmol}$ ) dissolved in $30 \mathrm{~mL}$ of anhydrous THF was added. The combined solution was then warmed to room temperature and left to stir for $24 \mathrm{~h}$. Solvent was then removed under reduced pressure and the product purified by column chromatography using $\mathrm{CH}_{2} \mathrm{Cl}_{2}$ as the eluent $\left(\mathrm{R}_{\mathrm{f}}=1.00\right)$. The product was isolated as a white crystalline solid (324mg, 82\%). ${ }^{1} \mathrm{H}$ NMR $\left(\mathrm{CDCl}_{3}, 300 \mathrm{MHz}\right) \delta 7.64(2 \mathrm{H}, \mathrm{s}$, aromatic $H), 7.36(2 \mathrm{H}, \mathrm{dd}$, $\left.{ }^{3} J_{\mathrm{HH}}=3.85 \mathrm{~Hz},{ }^{3} J_{\mathrm{HH}}=2.49 \mathrm{~Hz}\right), 7.10(4 \mathrm{H}, \mathrm{m}$, aromatic $H), 6.94\left(2 \mathrm{H}, \mathrm{dd},{ }^{3} J_{\mathrm{HH}}=17.47\right.$ $\mathrm{Hz},{ }^{3} J_{\mathrm{HH}}=10.97 \mathrm{~Hz}$, vinylic $\left.H\right) 5.73\left(2 \mathrm{H}, \mathrm{dd},{ }^{3} J_{\mathrm{HH}}=17.46 \mathrm{~Hz},{ }^{3} J_{\mathrm{HH}}=1.19 \mathrm{~Hz}\right.$, vinylic $H), 5.27\left(2 \mathrm{H}, \mathrm{dd},{ }^{3} J_{\mathrm{HH}}=10.98,{ }^{3} J_{\mathrm{HH}}=1.18 \mathrm{~Hz}\right.$, vinylic $\left.H\right) .{ }^{13} \mathrm{C} \mathrm{NMR}\left(\mathrm{CD}_{2} \mathrm{Cl}_{2}, 100 \mathrm{MHz}\right)$ $\delta$ 142.0, 136.4, 135.7, 133.30, 128.9, 128.4, 127.9, 126.5, 116.4. EI-MS: $\mathrm{m} / \mathrm{z}=294\left(\mathrm{M}^{+}\right.$, $100 \%)$. UV-vis $\left(\mathrm{CH}_{2} \mathrm{Cl}_{2}\right): \lambda_{\max }(\varepsilon)=276(33200) \mathrm{nm}\left(\mathrm{L} \mathrm{mol}^{-1} \mathrm{~cm}^{-1}\right)$. IR $(\mathrm{KBr}): v=2924$, 
2855, 1419, 1232, 1211, 1042, 985, 915, 898, 851, 836, 707, $699 \mathrm{~cm}^{-1} . \mathrm{Mp} .108-110^{\circ} \mathrm{C}$. Anal. Calcd for $\mathrm{C}_{18} \mathrm{H}_{14} \mathrm{~S}_{2}$ : C, 73.43; H, 4.79. Found: C, 73.67, H, 5.10.

Synthesis of 2,5-di(5-methyl-2-thienyl)-1,4-divinylbenzene (4b). The same procedure was used as for preparation of $\mathbf{4 a}$. $\mathrm{R}_{\mathrm{f}}=1\left(\mathrm{CH}_{2} \mathrm{Cl}_{2}\right)$. The product was isolated as a white solid (yield 76\%). ${ }^{1} \mathrm{H}$ NMR $\left(\mathrm{CD}_{2} \mathrm{Cl}_{2}, 400 \mathrm{MHz}\right) \delta 7.61(2 \mathrm{H}, \mathrm{s}$, aromatic $H), 7.00(2 \mathrm{H}$, dd, ${ }^{3} J_{\mathrm{HH}}=17.47 \mathrm{~Hz},{ }^{3} J_{\mathrm{HH}}=10.98 \mathrm{~Hz}$, vinylic $\left.H\right), 6.90\left(2 \mathrm{H}, \mathrm{d},{ }^{3} J_{\mathrm{HH}}=3.45 \mathrm{~Hz}\right.$, aromatic $\left.H\right)$, $6.78\left(2 \mathrm{H}, \mathrm{dd},{ }^{3} J_{\mathrm{HH}}=3.43 \mathrm{~Hz},{ }^{3} J_{\mathrm{HH}}=1.07 \mathrm{~Hz}\right.$, aromatic H), $5.74\left(2 \mathrm{H}, \mathrm{dd},{ }^{3} J_{\mathrm{HH}}=17.45\right.$ $\mathrm{Hz},{ }^{3} J_{\mathrm{HH}}=1.19 \mathrm{~Hz}$, vinylic $\left.H\right), 5.28\left(2 \mathrm{H}, \mathrm{dd},{ }^{3} J_{\mathrm{HH}}=10.96,{ }^{3} J_{\mathrm{HH}}=1.17 \mathrm{~Hz}\right.$, vinylic $\left.H\right)$, $2.53\left(6 \mathrm{H}, \mathrm{s}, \mathrm{CH}_{3}\right) .{ }^{13} \mathrm{C} \mathrm{NMR}\left(\mathrm{CD}_{2} \mathrm{Cl}_{2}, 100 \mathrm{MHz}\right) \delta 141.3,139.7,136.1,135.9,133.3$, 128.7, 128.25, 126.2, 116.0, 15.6. EI-MS: $\mathrm{m} / \mathrm{z}=322.1\left(\mathrm{M}^{+}, 100 \%\right)$. UV-vis $\left(\mathrm{CH}_{2} \mathrm{Cl}_{2}\right)$ : $\lambda_{\max }(\varepsilon)=279(23600) \mathrm{nm}\left(\mathrm{L} \mathrm{mol}^{-1} \mathrm{~cm}^{-1}\right)$. IR $(\mathrm{KBr}): v=2924,2855,1376,1260,1240$, 1212, 1093, 1060, 993, 897, 803, 794, $617 \mathrm{~cm}^{-1}$. Mp. 115-117 ${ }^{\circ}$. Anal. Calcd for $\mathrm{C}_{20} \mathrm{H}_{18} \mathrm{~S}_{2}$ : C, 74.49; H, 5.63. Found: C, 74.33; H, 5.55.

Synthesis of 2,5-di(5-hexyl-2-thienyl)-1,4-divinylbenzene (4c). The same procedure was used as for preparation of $\mathbf{4 a}$. The crude product was chromatographed on silica using a $\mathrm{CH}_{2} \mathrm{Cl}_{2} /$ hexanes mixture (1:1) $\left(\mathrm{R}_{\mathrm{f}}=0.96\right)$ and isolated as a white solid (yield 74\%). ${ }^{1} \mathrm{H}$ NMR $\left(\mathrm{CD}_{2} \mathrm{Cl}_{2}, 400 \mathrm{MHz}\right) \delta 7.61(2 \mathrm{H}, \mathrm{s}$, aromatic $H), 7.01\left(2 \mathrm{H}, \mathrm{dd},{ }^{3} J_{\mathrm{HH}}=17.47\right.$ $\mathrm{Hz},{ }^{3} J_{\mathrm{HH}}=10.98 \mathrm{~Hz}$, vinylic $\left.H\right), 6.91\left(2 \mathrm{H}, \mathrm{d},{ }^{3} J_{\mathrm{HH}}=3.48 \mathrm{~Hz}\right.$, aromatic $\left.H\right), 6.79(2 \mathrm{H}, \mathrm{d}$, ${ }^{3} J_{\mathrm{HH}}=3.48 \mathrm{~Hz}$, aromatic $\left.H\right), 5.74\left(2 \mathrm{H}, \mathrm{dd},{ }^{3} J_{\mathrm{HH}}=17.44 \mathrm{~Hz},{ }^{3} J_{\mathrm{HH}}=1.11 \mathrm{~Hz}\right.$, vinylic $\left.H\right)$, $5.28\left(2 \mathrm{H}, \mathrm{dd},{ }^{3} J_{\mathrm{HH}}=10.97,{ }^{3} J_{\mathrm{HH}}=1.13 \mathrm{~Hz}\right.$, vinylic $\left.H\right), 2.85\left(4 \mathrm{H}, \mathrm{t},{ }^{3} J_{\mathrm{HH}}=7.63 \mathrm{~Hz}, \mathrm{CH}_{2}\right)$, 1.76-1.68 (4H, m, CH$), 1.51-1.32\left(12 \mathrm{H}, \mathrm{m}, \mathrm{CH}_{2}\right), 0.89\left(6 \mathrm{H}, \mathrm{t},{ }^{3} \mathrm{~J}_{\mathrm{HH}}=6.98 \mathrm{~Hz}, \mathrm{CH}_{3}\right) .{ }^{13} \mathrm{C}$ NMR $\left(\mathrm{CD}_{2} \mathrm{Cl}_{2}, 100 \mathrm{MHz}\right) \delta 147.5,139.4,136.1,136.0,133.4,128.7,128.0,124.9,116.0$, 32.3, 32.2, 30.7, 29.4, 23.2, 14.4. EI-MS: $\mathrm{m} / \mathrm{z}=462.2\left(\mathrm{M}^{+}, 100 \%\right)$. UV-vis $\left(\mathrm{CH}_{2} \mathrm{Cl}_{2}\right): \lambda_{\max }$ $(\varepsilon)=280(30900) \mathrm{nm}\left(\mathrm{L} \mathrm{mol}^{-1} \mathrm{~cm}^{-1}\right)$. IR $(\mathrm{KBr}): v=2958,2925,2849,1493,1473,1271$, $1055,1038,996,957,913,903,801,607,517 \mathrm{~cm}^{-1}$. Mp. $50^{\circ} \mathrm{C}$. Anal. Calcd for $\mathrm{C}_{30} \mathrm{H}_{38} \mathrm{~S}_{2}$ : C, 77.86; H, 8.28. Found: C, 77.65; H, 8.33.

Synthesis of 2,5-bis(5-dodecyl-2-thienyl)-1,4-divinylbenzene (4d). The same procedure was used as for preparation of $4 \mathbf{c}$. $\mathrm{R}_{\mathrm{f}}=0.96$ (hexane $/ \mathrm{CH}_{2} \mathrm{Cl}_{2}(1: 1)$ ). The product was isolated as a white solid (yield $80 \%)$. ${ }^{1} \mathrm{H}$ NMR $\left(\mathrm{CD}_{2} \mathrm{Cl}_{2}, 400 \mathrm{MHz}\right) \delta 7.61(2 \mathrm{H}$, s, aromatic $H), 7.01\left(2 \mathrm{H}, \mathrm{dd},{ }^{3} J_{\mathrm{HH}}=17.44 \mathrm{~Hz},{ }^{3} J_{\mathrm{HH}}=10.98 \mathrm{~Hz}\right.$, vinylic $\left.H\right), 6.91(2 \mathrm{H}, \mathrm{d}$, ${ }^{3} J_{\mathrm{HH}}=3.46 \mathrm{~Hz}$, aromatic $\left.H\right), 6.79\left(2 \mathrm{H}, \mathrm{d},{ }^{3} J_{\mathrm{HH}}=3.44 \mathrm{~Hz}\right.$, aromatic $\left.H\right), 5.74\left(2 \mathrm{H}, \mathrm{d},{ }^{3} J_{\mathrm{HH}}\right.$ $=17.44 \mathrm{~Hz}$, vinylic $H), 5.28\left(2 \mathrm{H}, \mathrm{d},{ }^{3} J_{\mathrm{HH}}=11.04\right.$, vinylic $\left.H\right), 2.84\left(4 \mathrm{H}, \mathrm{t},{ }^{3} J_{\mathrm{HH}}=7.52 \mathrm{~Hz}\right.$, $\left.\mathrm{CH}_{2}\right), 1.75-1.68\left(4 \mathrm{H}, \mathrm{m}, \mathrm{CH}_{2}\right), 1.41-1.27\left(36 \mathrm{H}, \mathrm{m}, \mathrm{CH}_{2}\right), 0.88\left(6 \mathrm{H}, \mathrm{t},{ }^{3} J_{\mathrm{HH}}=6.30 \mathrm{~Hz}\right.$, $\left.\mathrm{CH}_{3}\right) .{ }^{13} \mathrm{C} \mathrm{NMR}\left(\mathrm{CD}_{2} \mathrm{Cl}_{2}, 100 \mathrm{MHz}\right) \delta 147.5,139.3,136.1,133.4,128.7,128.0,124.9$, 116.0, 32.5, 32.4, 30.7, 30.3, 30.2, 30.1, 29.9, 29.7, 23.3, 14.4. EI-MS: $m / z=630.4\left(\mathrm{M}^{+}\right.$, $100 \%)$ UV-vis $\left(\mathrm{CH}_{2} \mathrm{Cl}_{2}\right): \lambda_{\max }(\varepsilon)=280(31600) \mathrm{nm}\left(\mathrm{L} \mathrm{mol}^{-1} \mathrm{~cm}^{-1}\right)$. IR $(\mathrm{KBr}): v=2960$, 2916, 2849, 1497, 1472, 1465, 1058, 1005, 960, 907, 898, 808, 718, 605, 523 $\mathrm{cm}^{-1}$. Mp. $81^{\circ} \mathrm{C}$. Anal. Calcd for $\mathrm{C}_{42} \mathrm{H}_{62} \mathrm{~S}_{2}$ : C, 79.94; H, 9.90. Found: C, 79.88; H, 10.08 .

Synthesis of anthra(1,2-b: 5,6-b')dithiophene (5a). A $500 \mathrm{~mL}$ quartz flask was charged with 2,5-bis(2-thienyl)-1,4-divinylbenzene $4 a(90 \mathrm{mg}, 0.31 \mathrm{mmol})$, iodine $(154 \mathrm{mg}$, $0.61 \mathrm{mmol}$ ), and $300 \mathrm{~mL}$ of degassed benzene. The flask was irradiated with UV light while the progress of the reaction was monitored by UV-vis spectroscopy. Once complete 
the organic solution was first washed with an aqueous sodium thiosulfate solution, then water, and dried over $\mathrm{Na}_{2} \mathrm{SO}_{4}$. After filtration, the solvent was removed under reduced pressure to yield a dark brown solid, which was chromatographed on silica using a hexane $/ \mathrm{CH}_{2} \mathrm{Cl}_{2}(1: 1)\left(\mathrm{R}_{\mathrm{f}}=0.64\right)$ mixture as the eluent. The product was isolated as a yellow solid and recrystallized in $\mathrm{CH}_{2} \mathrm{Cl}_{2}$ at $-10{ }^{\circ} \mathrm{C}(36 \mathrm{mg}, 40 \%)$. ${ }^{1} \mathrm{H} \mathrm{NMR}\left(\mathrm{CD}_{2} \mathrm{Cl}_{2}\right.$, $400 \mathrm{MHz}) \delta 8.76(2 \mathrm{H}, \mathrm{s}$, aromatic $H), 7.94\left(2 \mathrm{H}, \mathrm{d},{ }^{3} J_{\mathrm{HH}}=8.82 \mathrm{~Hz}\right.$, aromatic $\left.H\right), 7.87(2 \mathrm{H}$, $\mathrm{d},{ }^{3} J_{\mathrm{HH}}=8.80 \mathrm{~Hz}$, aromatic $\left.H\right), 7.60\left(2 \mathrm{H}, \mathrm{d},{ }^{3} J_{\mathrm{HH}}=5.22 \mathrm{~Hz}\right.$, aromatic $\left.H\right), 7.53(2 \mathrm{H}, \mathrm{d}$, ${ }^{3} J_{\mathrm{HH}}=5.22 \mathrm{~Hz}$, aromatic $\left.H\right) .{ }^{13} \mathrm{C} \mathrm{NMR}\left(\mathrm{CD}_{2} \mathrm{Cl}_{2}, 100 \mathrm{MHz}\right) \delta 137.6,137.6,130.5,127.8$, 126.1, 125.9, 125.7, 123.1, 123.1. EI-MS: $\mathrm{m} / \mathrm{z}=290\left(\mathrm{M}^{+}, 100 \%\right)$. UV-vis $\left(\mathrm{CH}_{2} \mathrm{Cl}_{2}\right): \lambda_{\max }$ $(\varepsilon)=304$ (142900), $292(60000) \mathrm{nm}\left(\mathrm{L} \mathrm{mol}^{-1} \mathrm{~cm}^{-1}\right)$. IR $(\mathrm{KBr}): v=2923,2855,1279$, $1145,1083,883,839,782,693,601 \mathrm{~cm}^{-1}$. Mp. $223-225^{\circ} \mathrm{C}$. Anal. Calcd for $\mathrm{C}_{18} \mathrm{H}_{10} \mathrm{~S}_{2}$ : C, 74.45; H, 3.47. Found: C, 74.80, H, 3.82. Fluorescence: $\Phi_{\mathrm{f}}=0.36$.

Synthesis of anthra(1,2-b: 5,6-b')di-5-methylthiophene (5b). The same procedure was used as for preparation of $5 \mathbf{a}$. $\mathrm{R}_{\mathrm{f}}=0.64$ (hexane $/ \mathrm{CH}_{2} \mathrm{Cl}_{2}(1: 1)$ ). The product was isolated as a yellow solid and recrystallized in $\mathrm{CH}_{2} \mathrm{Cl}_{2}$ at $-10{ }^{\circ} \mathrm{C}$ (yield $\left.37 \%\right)$. ${ }^{1} \mathrm{H} \mathrm{NMR}\left(\mathrm{CD}_{2} \mathrm{Cl}_{2}\right.$, 400MHz) $\delta 8.59(2 \mathrm{H}, \mathrm{s}$, aromatic $H), 7.86\left(2 \mathrm{H}, \mathrm{d},{ }^{3} \mathrm{~J}_{\mathrm{HH}}=8.77 \mathrm{~Hz}\right.$, aromatic $\left.H\right), 7.72(2 \mathrm{H}$, $\mathrm{d},{ }^{3} \mathrm{~J}_{\mathrm{HH}}=8.78 \mathrm{~Hz}$, aromatic $\left.\mathrm{H}\right), 7.17(2 \mathrm{H}, \mathrm{s}$, aromatic $\mathrm{H}), 2.70\left(6 \mathrm{H}, \mathrm{s}, \mathrm{CH}_{3}\right) .{ }^{13} \mathrm{C} \mathrm{NMR}$ $\left(\mathrm{CD}_{2} \mathrm{Cl}_{2}, 100 \mathrm{MHz}\right) \delta 140.6,137.9,136.5,130.2,127.4,125.6,124.1,122.7,122.6,16.4$. EI-MS: $m / z=318\left(\mathrm{M}^{+}, 100 \%\right)$. UV-vis $\left(\mathrm{CH}_{2} \mathrm{Cl}_{2}\right): \lambda_{\max }(\varepsilon)=309$ (217900), $296(96300)$ $\mathrm{nm}\left(\mathrm{L} \mathrm{mol}^{-1} \mathrm{~cm}^{-1}\right)$. IR (KBr): $v=2921,2850,1458,1269,1201,1091,983,869,829,801$, $699,670,606,490 \mathrm{~cm}^{-1}$. Mp. $>230^{\circ} \mathrm{C}$. Fluorescence: $\Phi_{\mathrm{f}}=0.56$.

Synthesis of anthra(1,2-b: 5,6-b')di-5-hexylthiophene (5c). The same procedure was used as for preparation of $5 \mathrm{a} . \mathrm{R}_{\mathrm{f}}=0.8$ (hexane $/ \mathrm{CH}_{2} \mathrm{Cl}_{2}(1: 1)$ ). The product was isolated as a yellow solid and recrystallized in $\mathrm{CHCl}_{3}$ at $-10{ }^{\circ} \mathrm{C}$ (yield $62 \%$ ). ${ }^{1} \mathrm{H}$ NMR $\left(\mathrm{CD}_{2} \mathrm{Cl}_{2}\right.$, $400 \mathrm{MHz}) \delta 8.60(2 \mathrm{H}, \mathrm{s}$, aromatic $H), 7.86\left(2 \mathrm{H}, \mathrm{d},{ }^{3} J_{\mathrm{HH}}=8.80 \mathrm{~Hz}\right.$, aromatic $\left.H\right), 7.72(2 \mathrm{H}$, $\mathrm{d},{ }^{3} J_{\mathrm{HH}}=8.80 \mathrm{~Hz}$, aromatic $\left.H\right), 7.18(2 \mathrm{H}, \mathrm{s}$, aromatic $H), 3.01\left(4 \mathrm{H}, \mathrm{t},{ }^{3} J_{\mathrm{HH}}=7.49 \mathrm{~Hz}\right.$, $\left.\mathrm{CH}_{2}\right), 1.90-1.78\left(4 \mathrm{H}, \mathrm{m}, \mathrm{CH}_{2}\right), 1.47-1.27\left(12 \mathrm{H}, \mathrm{m}, \mathrm{CH}_{2}\right), 0.91\left(6 \mathrm{H}, \mathrm{t},{ }^{3} J_{\mathrm{HH}}=6.93 \mathrm{~Hz}\right.$, $\left.\mathrm{CH}_{3}\right) .{ }^{13} \mathrm{C} \mathrm{NMR}\left(\mathrm{CD}_{2} \mathrm{Cl}_{2}, 100 \mathrm{MHz}\right) \delta 146.7,137.6,136.1,130.1,127.4,125.6,122.9$, 122.7, 122.6, 32.2, 32.1, 31.3, 29.4, 23.2, and 14.4. EI-MS: $m / z=458\left(\mathrm{M}^{+}, 100 \%\right)$. UVvis $\left(\mathrm{CH}_{2} \mathrm{Cl}_{2}\right): \lambda_{\max }(\varepsilon)=311(129500), 298(52900) \mathrm{nm}\left(\mathrm{L} \mathrm{mol}^{-1} \mathrm{~cm}^{-1}\right)$. IR $(\mathrm{KBr}): v=$ 2953, 2926, 2849, 1466, 1092, 984, 872, 832, 794, 724, 699, 670, 607, 491, $458 \mathrm{~cm}^{-1}$. Mp. $117-119^{\circ}$ C. Anal. Calcd for $\mathrm{C}_{30} \mathrm{H}_{34} \mathrm{~S}_{2}$ : C, 78.55; H, 7.47. Found: C, 78.28; H, 7.77. Fluorescence : $\Phi_{\mathrm{f}}=0.60$.

Anthra(1,2-b: 5,6-b')di-5-dodecylthiophene (5d). The same procedure was used as for preparation of 5a. $\mathrm{R}_{\mathrm{f}}=0.88$ (hexane $/ \mathrm{CH}_{2} \mathrm{Cl}_{2}(1: 1)$ ). The product was isolated as a yellow solid and recrystallized in $\mathrm{CHCl}_{3}$ at $-10{ }^{\circ} \mathrm{C}$ (yield $56 \%$ ). ${ }^{1} \mathrm{H} \mathrm{NMR}\left(\mathrm{CD}_{2} \mathrm{Cl}_{2}, 400 \mathrm{MHz}\right) \delta$ $8.61(2 \mathrm{H}, \mathrm{s}$, aromatic $H), 7.86\left(2 \mathrm{H}, \mathrm{d},{ }^{3} J_{\mathrm{HH}}=8.77 \mathrm{~Hz}\right.$, aromatic $\left.H\right), 7.73\left(2 \mathrm{H}, \mathrm{d},{ }^{3} J_{\mathrm{HH}}=\right.$ $8.79 \mathrm{~Hz}$, aromatic H), $7.19(2 \mathrm{H}$, s, aromatic $H), 3.01\left(4 \mathrm{H}, \mathrm{t},{ }^{3} J_{\mathrm{HH}}=7.49 \mathrm{~Hz}, \mathrm{CH}_{2}\right), 1.87-$ $1.80(4 \mathrm{H}, \mathrm{m} \mathrm{CH}), 1.50-1.27\left(36 \mathrm{H}, \mathrm{m}, \mathrm{CH}_{2}\right), 0.88\left(6 \mathrm{H}, \mathrm{t},{ }^{3} \mathrm{~J}_{\mathrm{HH}}=6.93 \mathrm{~Hz}, \mathrm{CH}_{3}\right) .{ }^{13} \mathrm{C}$ NMR $\left(\mathrm{CD}_{2} \mathrm{Cl}_{2}, 100 \mathrm{MHz}\right) \delta 146.7,137.7,136.2,130.2,127.5,125.6,122.9,122.8,122.6$, $32.5,32.2,31.3,30.3,30.2,30.1,30.0,29.9,29.7,23.3,14.5$. EI-MS: $m / z=626\left(\mathrm{M}^{+}\right.$, $100 \%)$. UV-vis $\left(\mathrm{CH}_{2} \mathrm{Cl}_{2}\right): \lambda_{\max }(\varepsilon)=311(162000), 298(67000) \mathrm{nm}\left(\mathrm{L} \mathrm{mol}^{-1} \mathrm{~cm}^{-1}\right)$. IR (KBr): v 2954, 2922, 2851, 1469, 1094, 984, 871, 826, 793, 720, 699, $606 \mathrm{~cm}^{-1}$. Mp. 123- 
$125^{\circ} \mathrm{C}$. Anal. Calcd for $\mathrm{C}_{42} \mathrm{H}_{58} \mathrm{~S}_{2}$ : C, 80.45; H, 9.32. Found: C, 80.10; H, 9.52. Fluorescence: $\Phi_{\mathrm{f}}=0.66$.
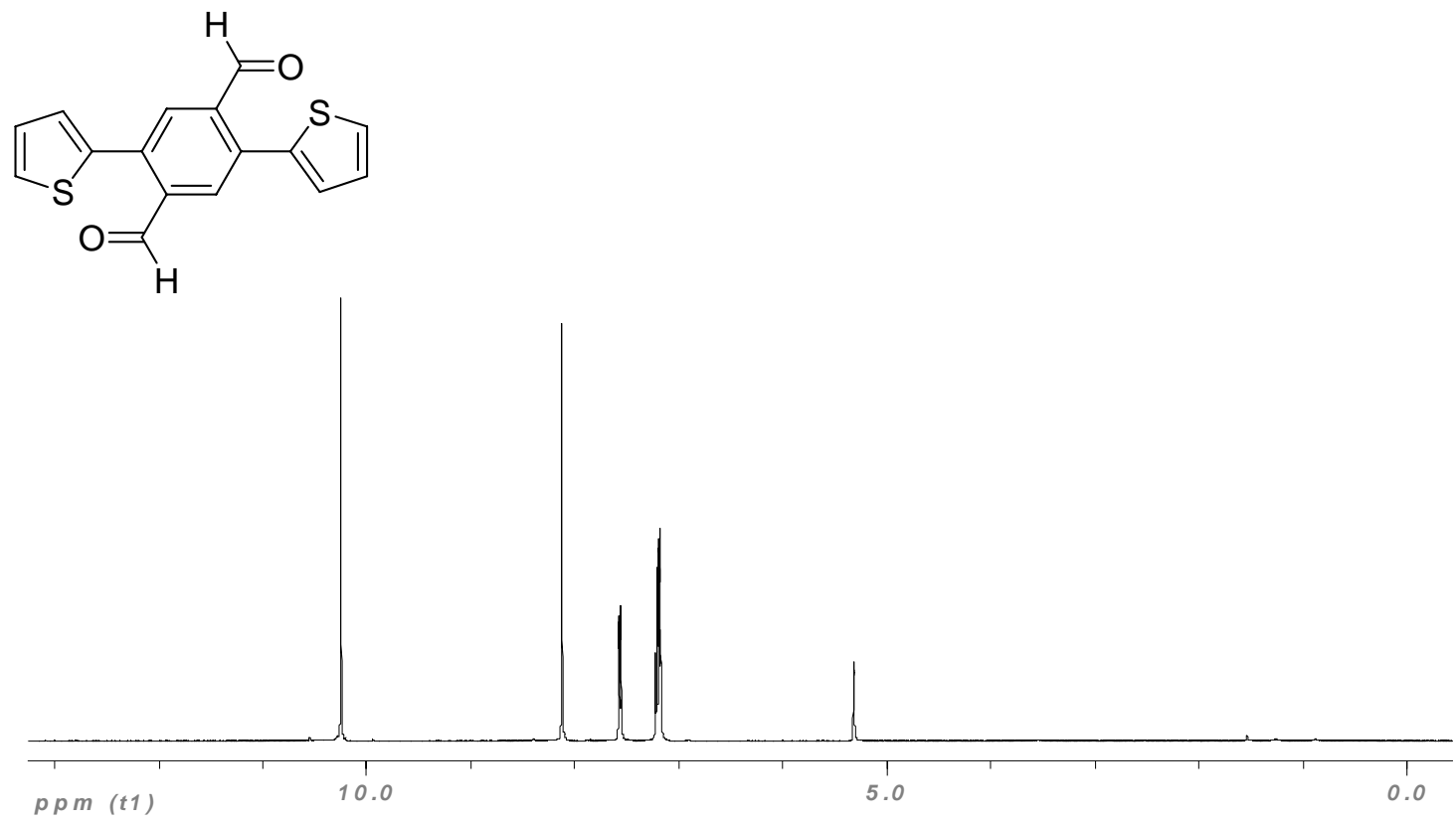

Figure 1. ${ }^{1} \mathrm{H}$ NMR spectrum of $3 \mathbf{a}\left(300 \mathrm{MHz}, \mathrm{CD}_{2} \mathrm{Cl}_{2}\right)$.
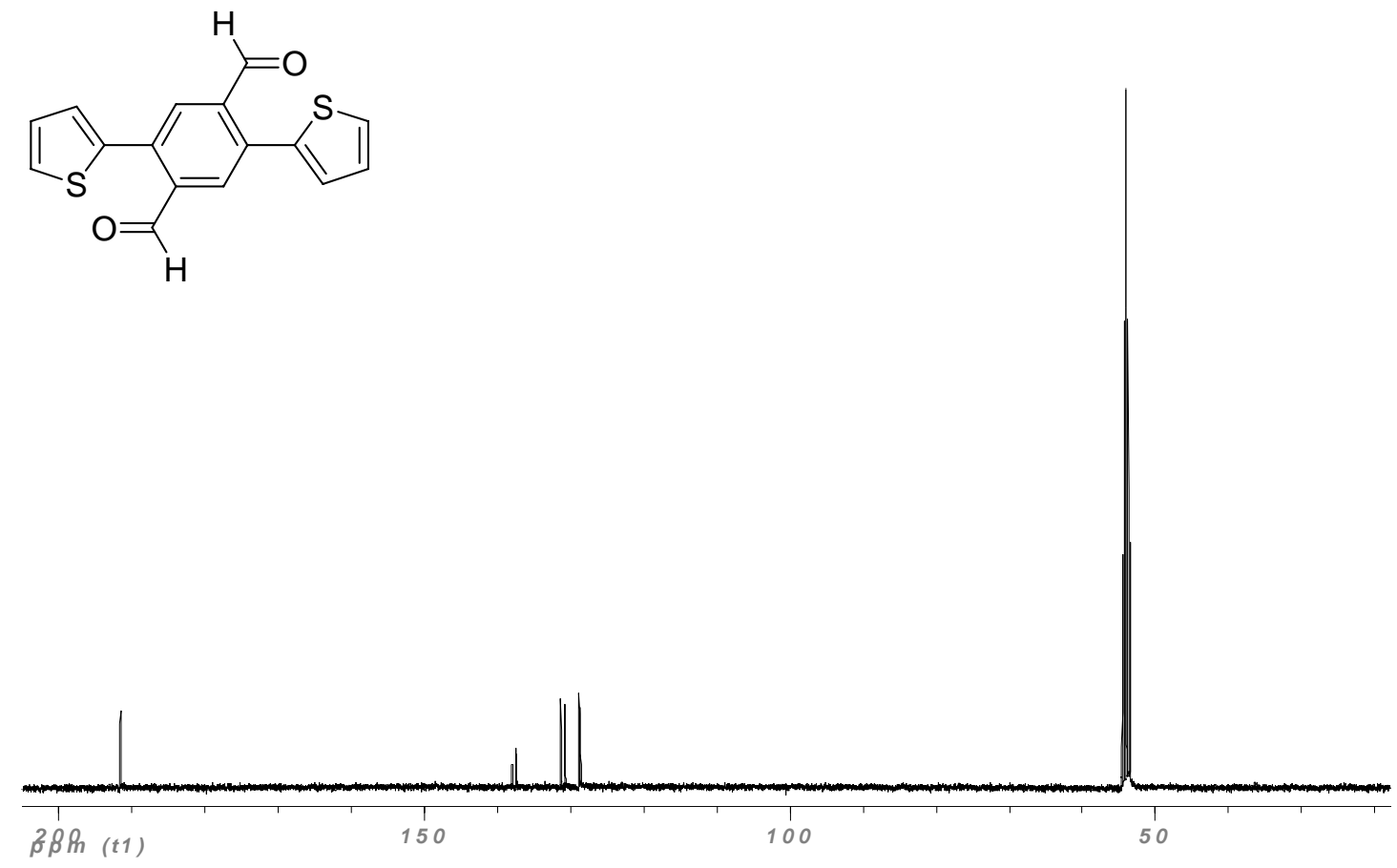

Figure 2. ${ }^{13} \mathrm{C}$ NMR spectrum of $3 \mathbf{a}\left(100 \mathrm{MHz}, \mathrm{CD}_{2} \mathrm{Cl}_{2}\right)$. 

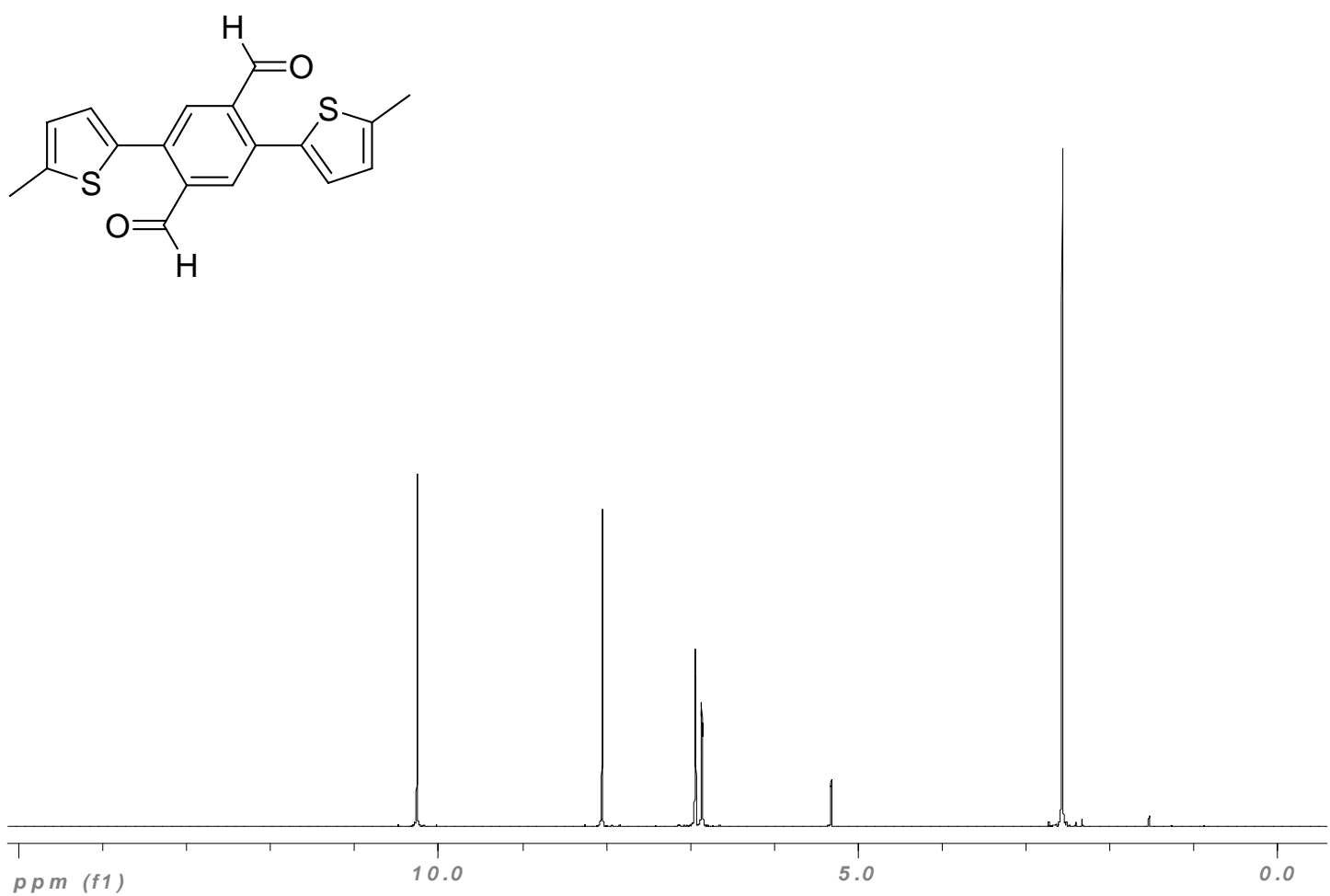

Figure 3. ${ }^{1} \mathrm{H}$ NMR spectrum of $3 \mathbf{b}\left(400 \mathrm{MHz}, \mathrm{CD}_{2} \mathrm{Cl}_{2}\right)$.
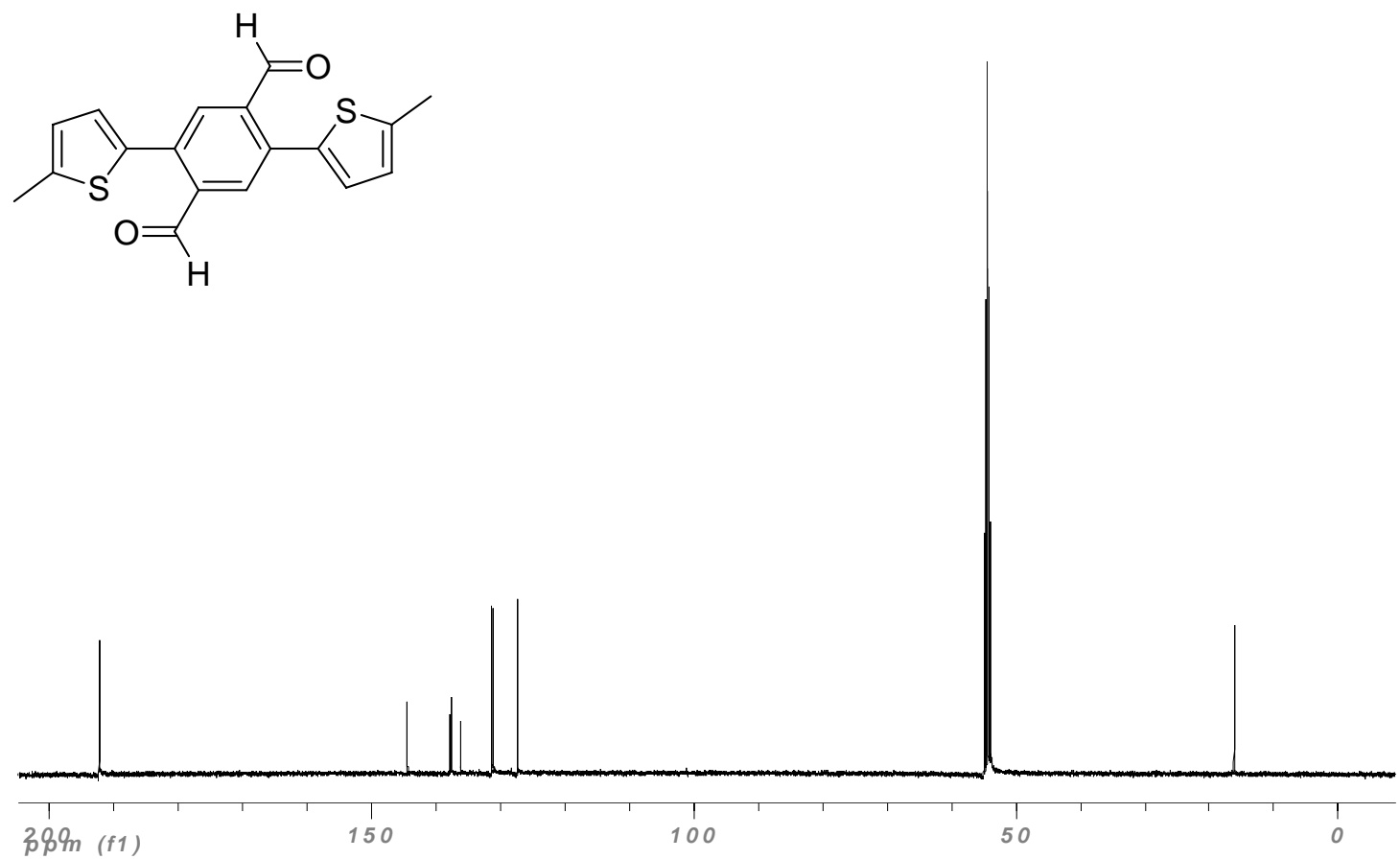

Figure 4. ${ }^{13} \mathrm{C}$ NMR spectrum of $\mathbf{3 b}\left(100 \mathrm{MHz}, \mathrm{CD}_{2} \mathrm{Cl}_{2}\right)$. 


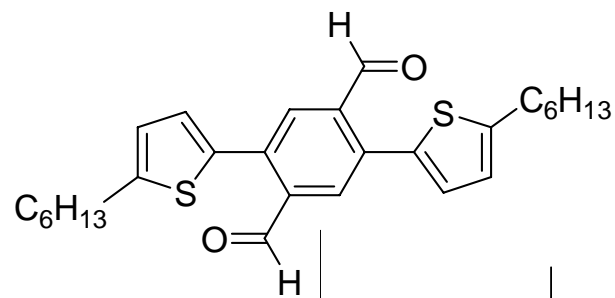

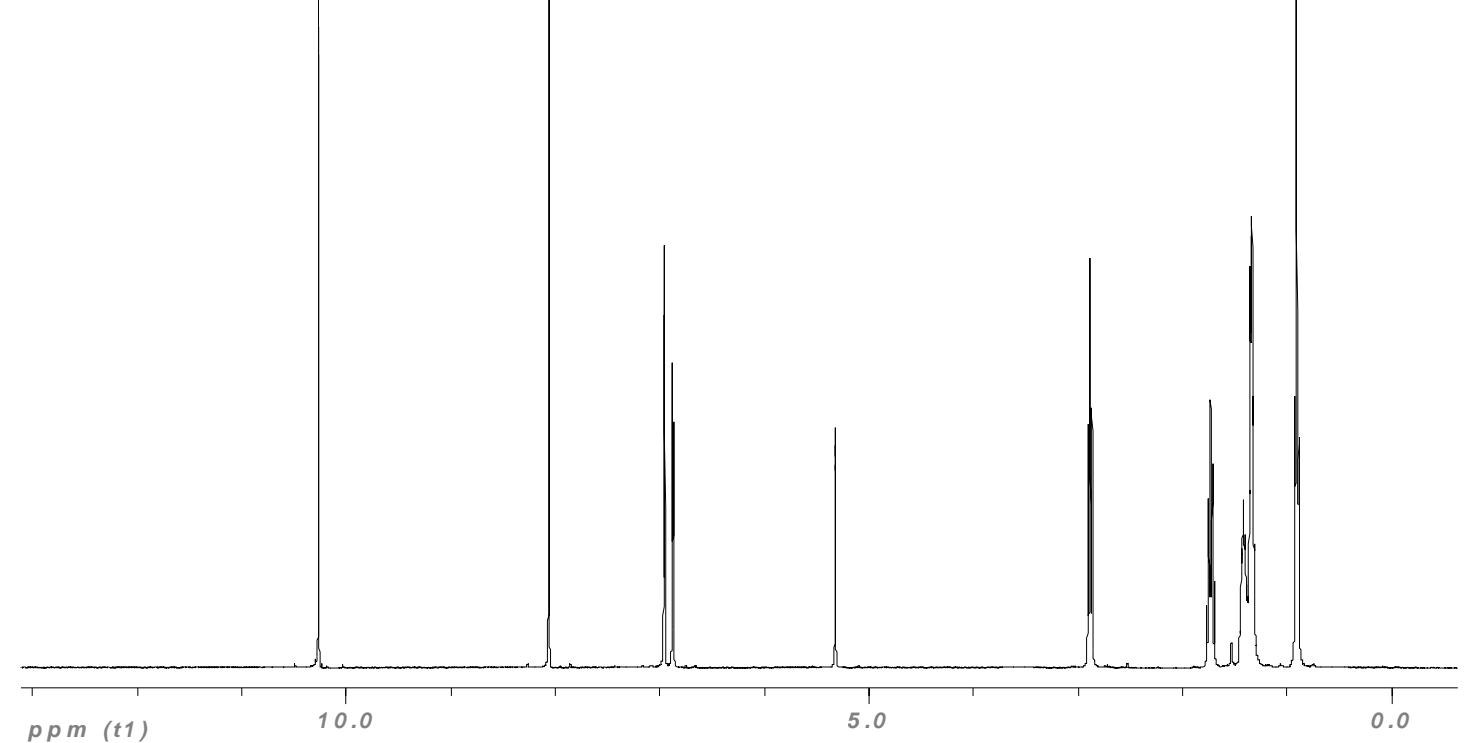

Figure 5. ${ }^{1} \mathrm{H}$ NMR spectrum of $3 \mathrm{c}\left(400 \mathrm{MHz}, \mathrm{CD}_{2} \mathrm{Cl}_{2}\right)$.

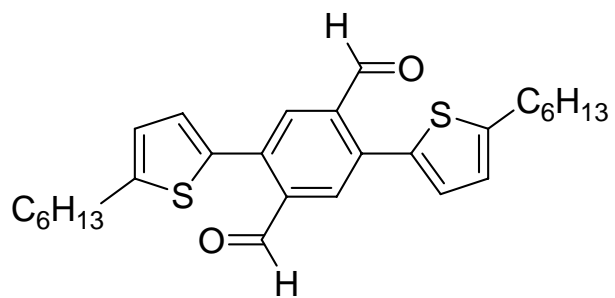

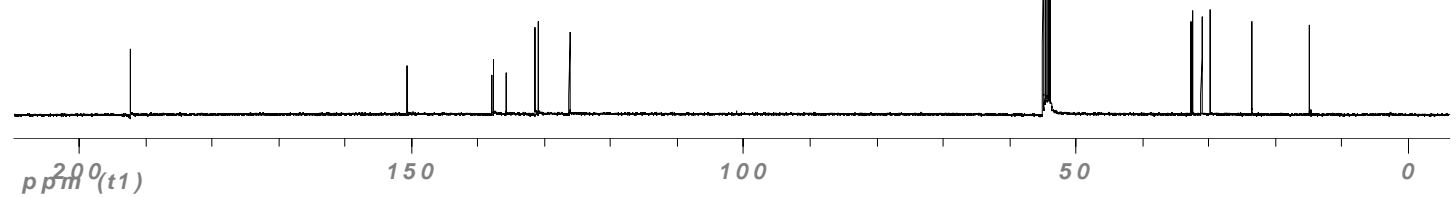

Figure 6. ${ }^{13} \mathrm{C}$ NMR spectrum of $3 \mathrm{c}\left(100 \mathrm{MHz}, \mathrm{CD}_{2} \mathrm{Cl}_{2}\right)$. 


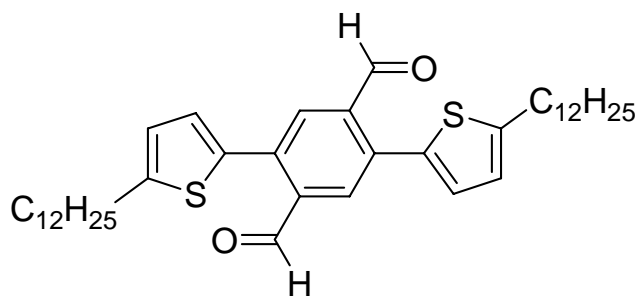

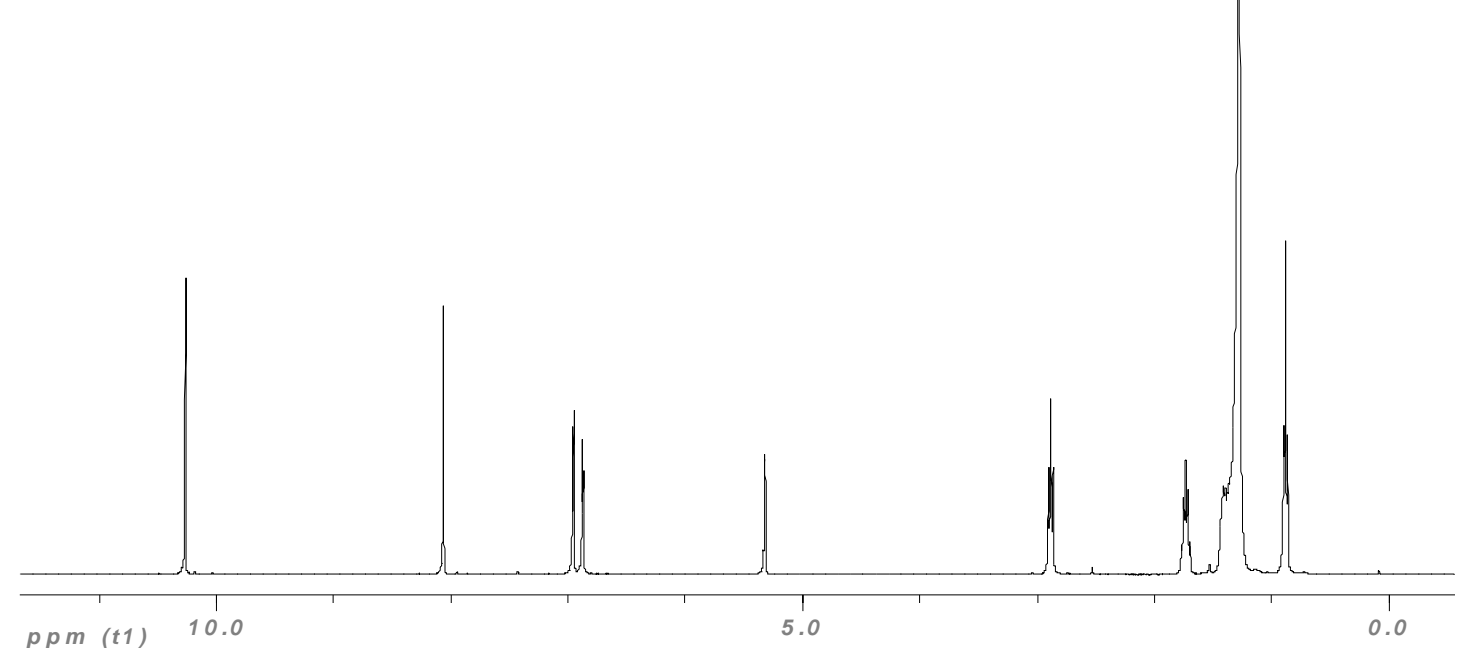

Figure $7 .{ }^{1} \mathrm{H} \mathrm{NMR}$ spectrum of $\mathbf{3 d}\left(400 \mathrm{MHz}, \mathrm{CD}_{2} \mathrm{Cl}_{2}\right)$.

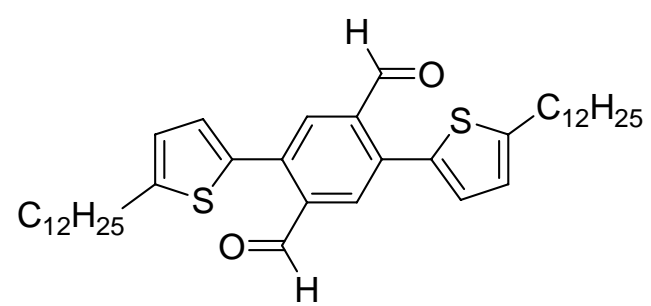

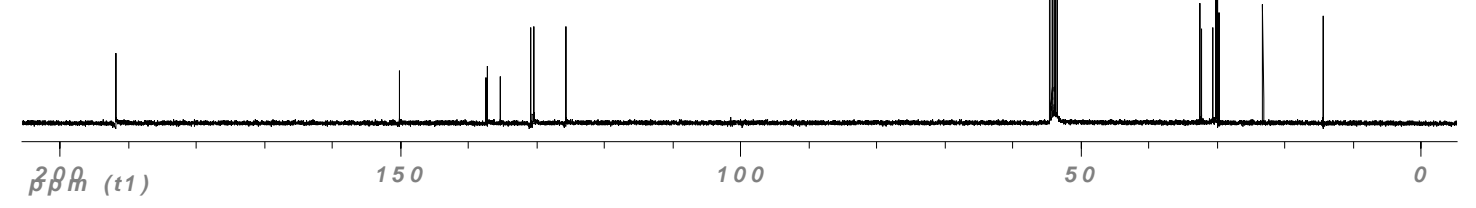

Figure 8. ${ }^{13} \mathrm{C}$ NMR spectrum of $3 d\left(100 \mathrm{MHz}, \mathrm{CD}_{2} \mathrm{Cl}_{2}\right)$. 


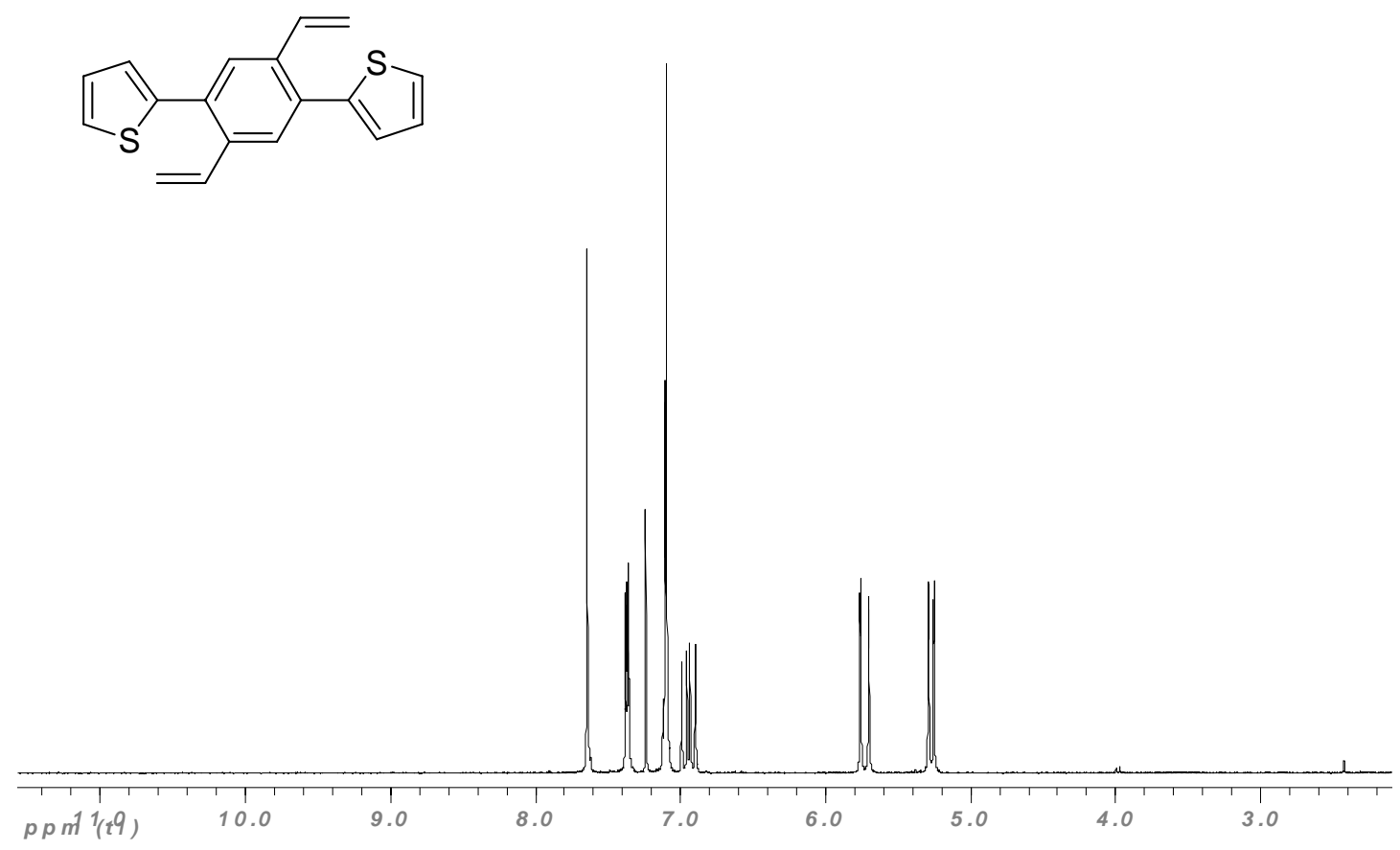

Figure 9. ${ }^{1} \mathrm{H} \mathrm{NMR}$ spectrum of $\mathbf{4 a}\left(300 \mathrm{MHz}, \mathrm{CDCl}_{3}\right)$.

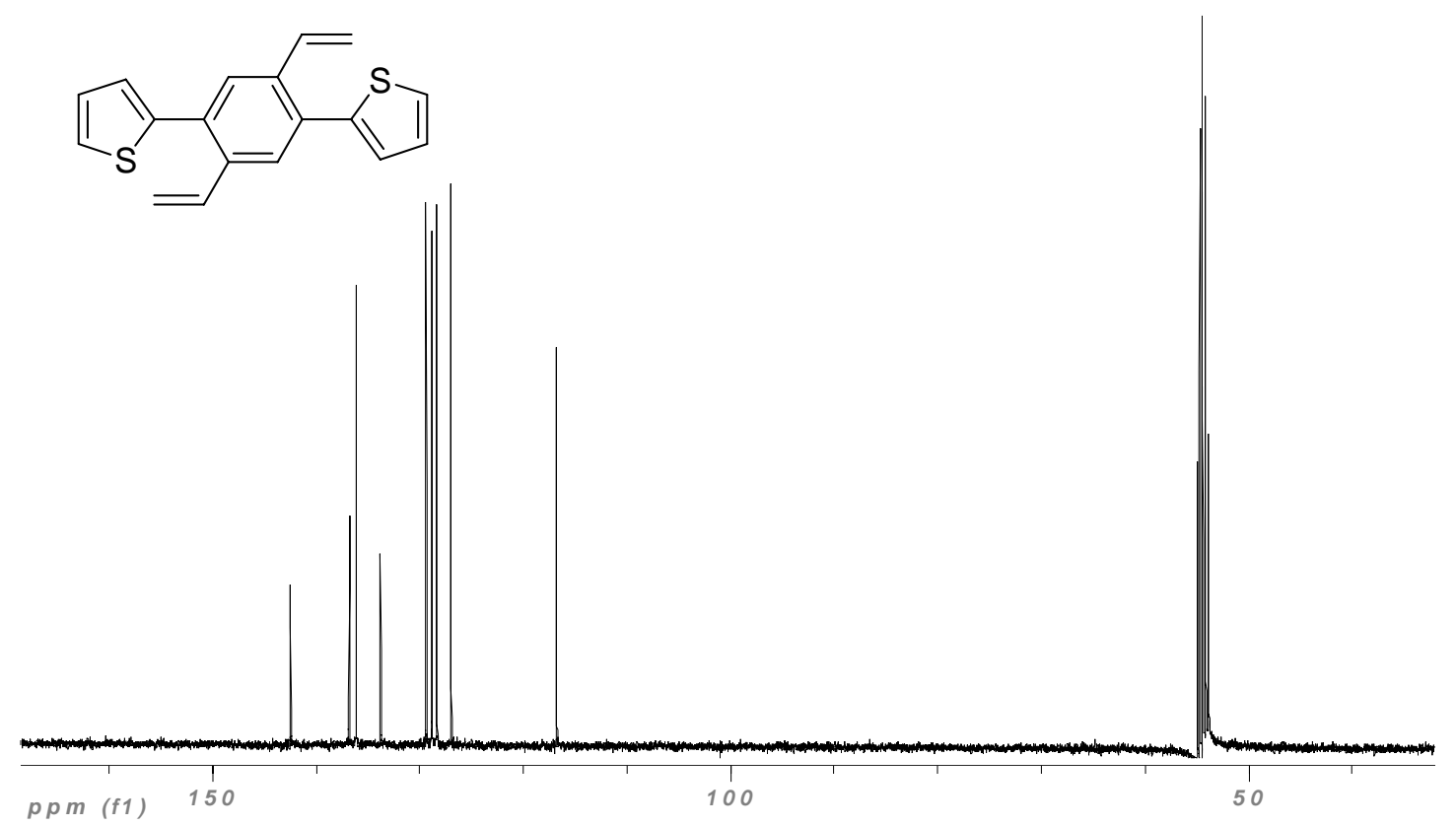

Figure 10. ${ }^{13} \mathrm{C}$ NMR spectrum of $4 a\left(100 \mathrm{MHz}, \mathrm{CD}_{2} \mathrm{Cl}_{2}\right)$. 

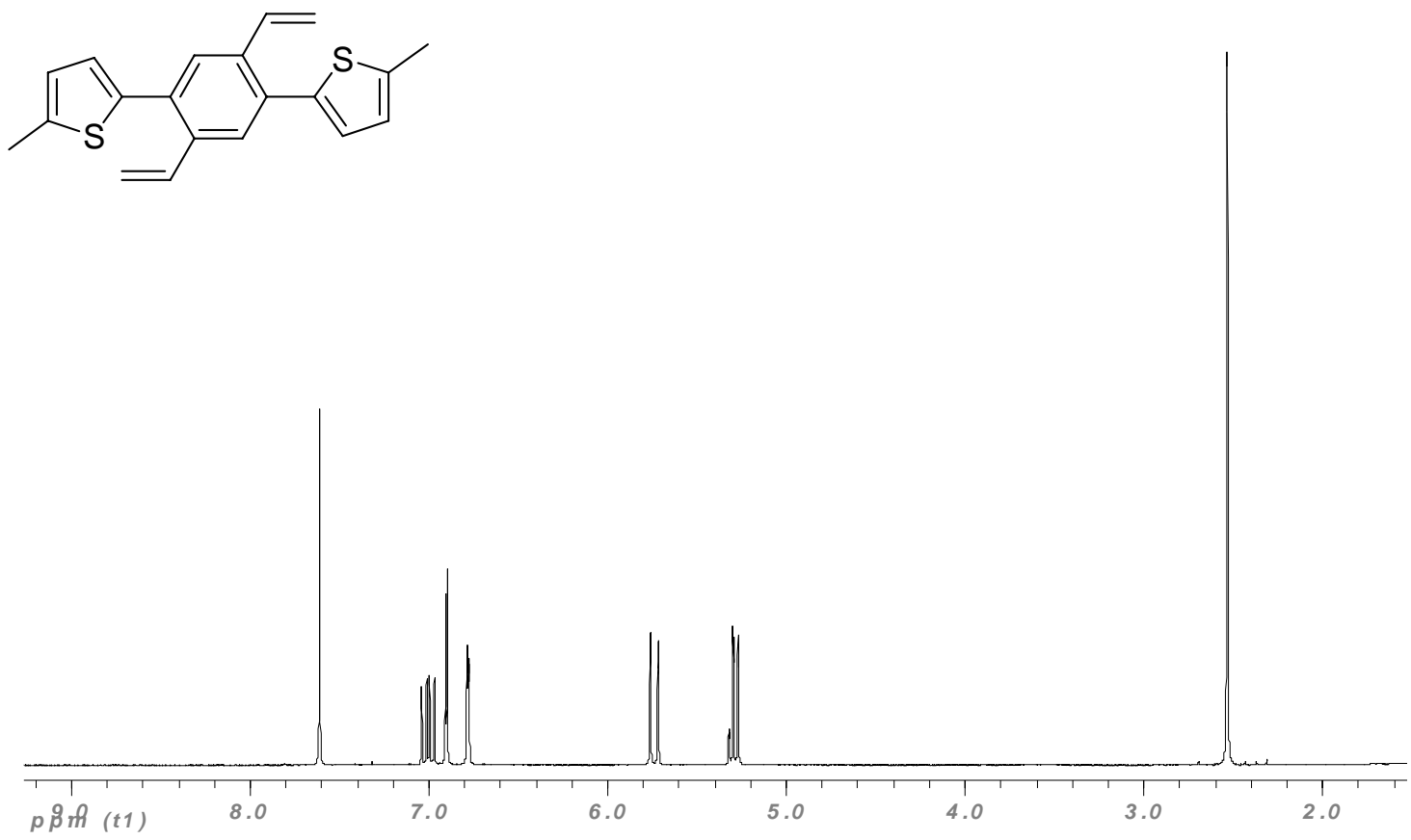

Figure 11. ${ }^{1} \mathrm{H}$ NMR spectrum of $\mathbf{4 b}\left(400 \mathrm{MHz}, \mathrm{CD}_{2} \mathrm{Cl}_{2}\right)$.
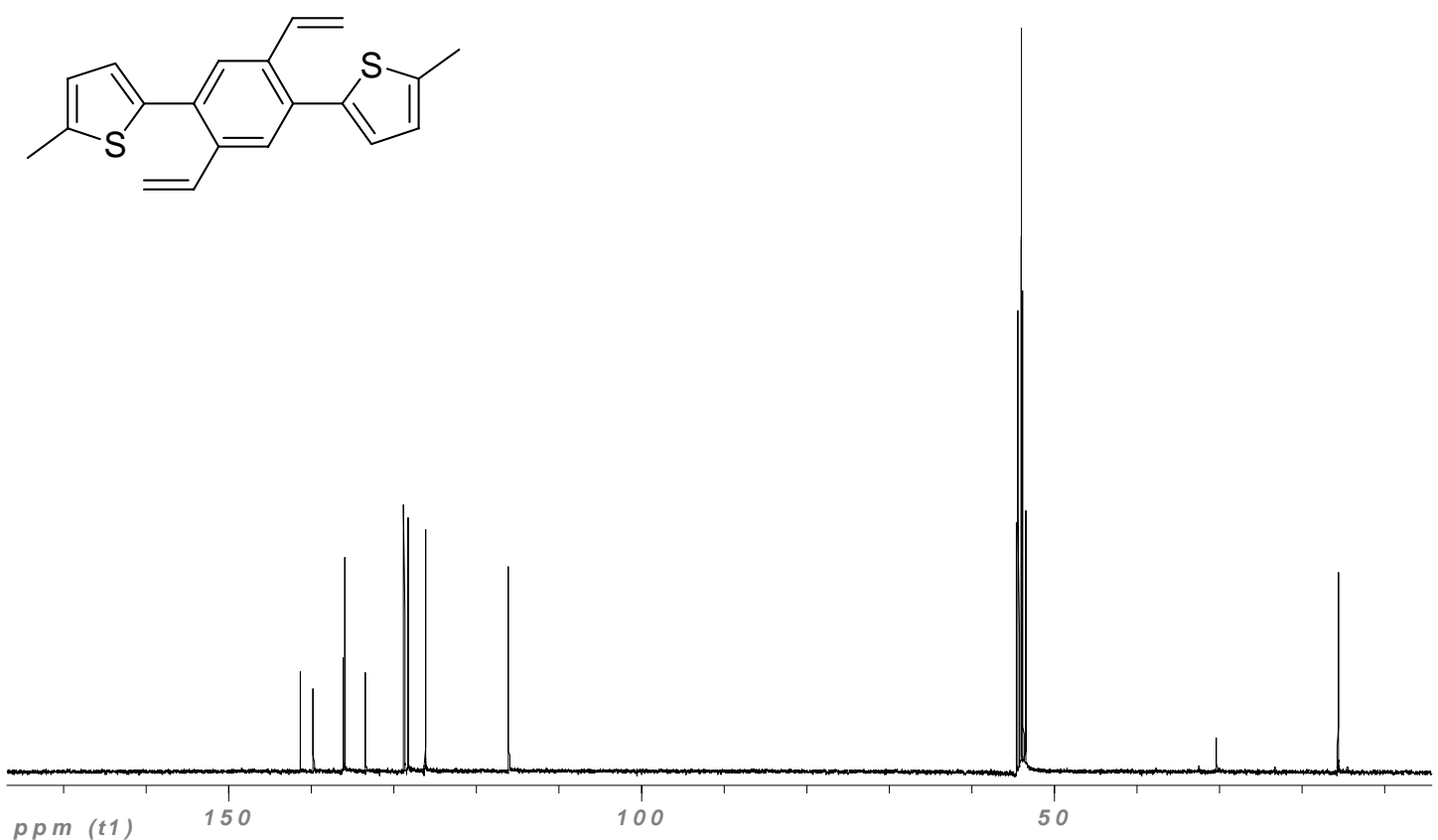

Figure 12. ${ }^{13} \mathrm{C}$ NMR spectrum of $\mathbf{4 b}\left(100 \mathrm{MHz}, \mathrm{CD}_{2} \mathrm{Cl}_{2}\right)$. 

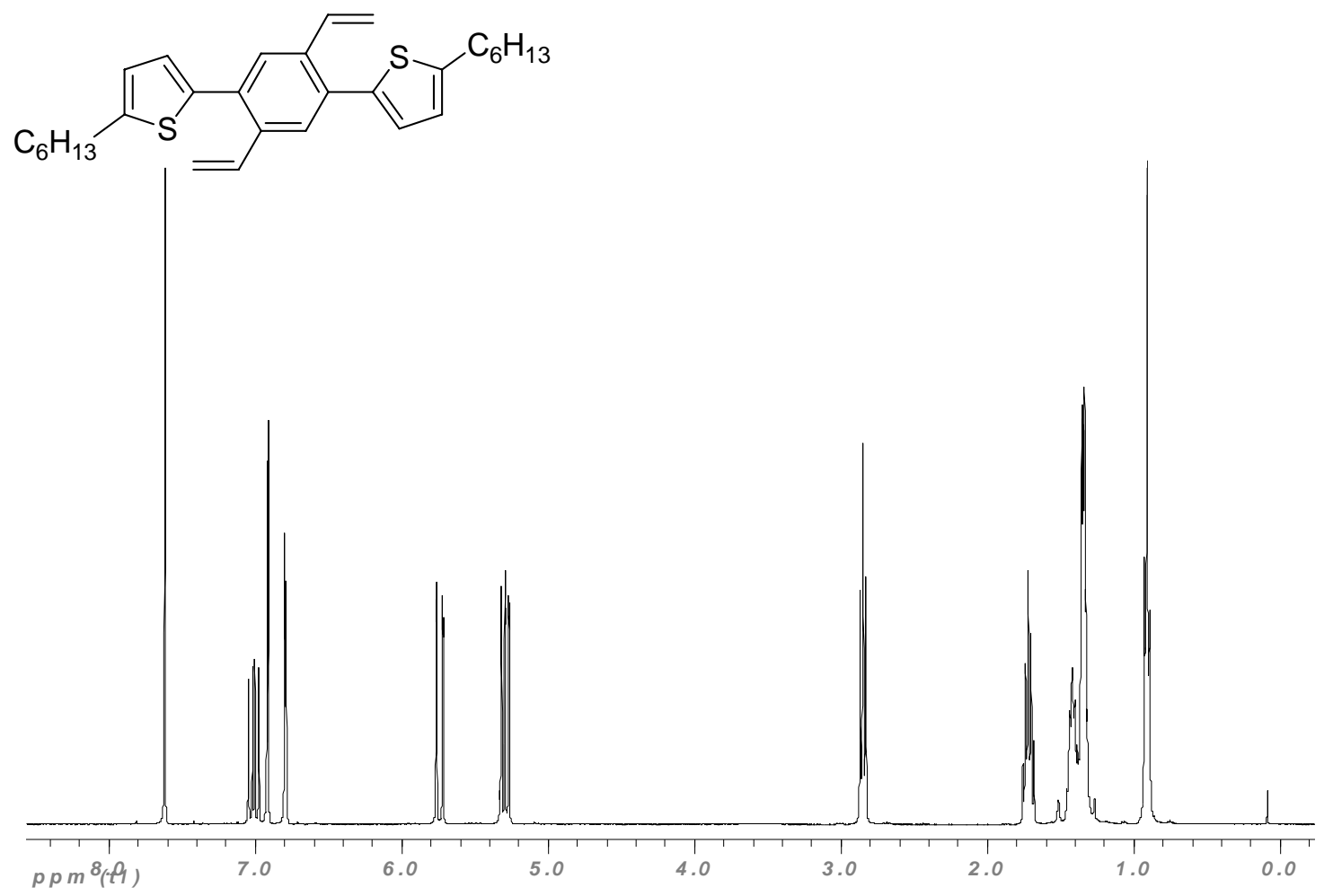

Figure 13. ${ }^{1} \mathrm{H}$ NMR spectrum of $4 \mathrm{c}\left(400 \mathrm{MHz}, \mathrm{CD}_{2} \mathrm{Cl}_{2}\right)$.

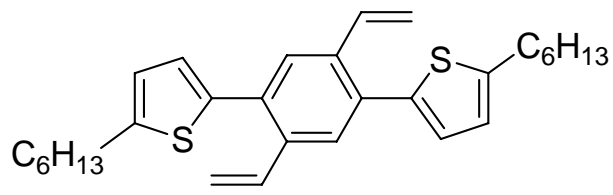

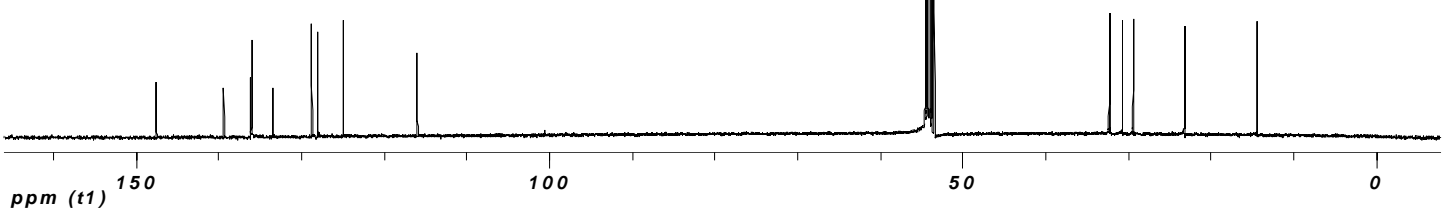

Figure 14. ${ }^{13} \mathrm{C}$ NMR spectrum of $4 \mathrm{c}\left(100 \mathrm{MHz}, \mathrm{CD}_{2} \mathrm{Cl}_{2}\right)$. 

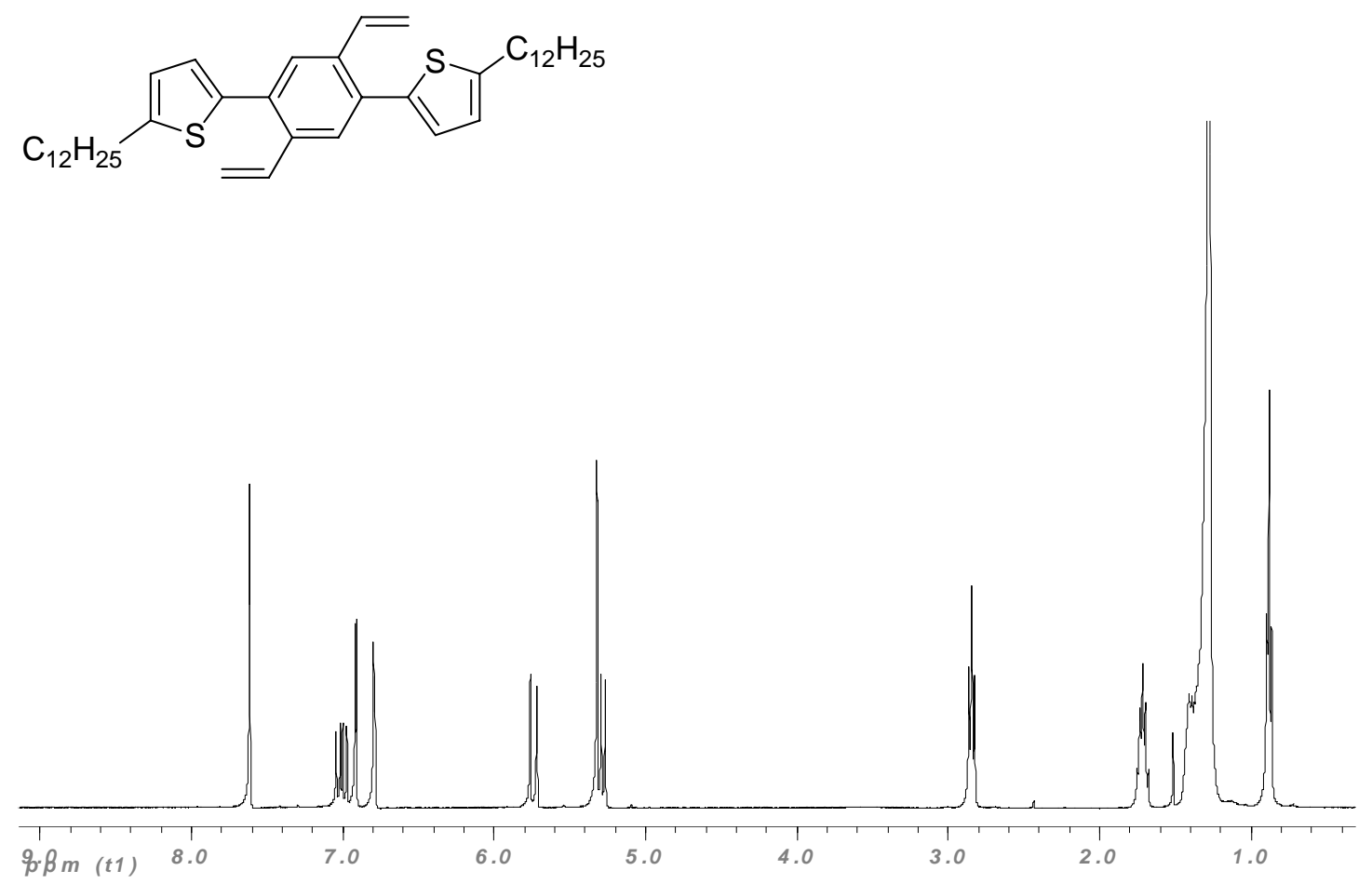

Figure 15. ${ }^{1} \mathrm{H}$ NMR spectrum of $\mathbf{4 d}\left(400 \mathrm{MHz}, \mathrm{CD}_{2} \mathrm{Cl}_{2}\right)$.
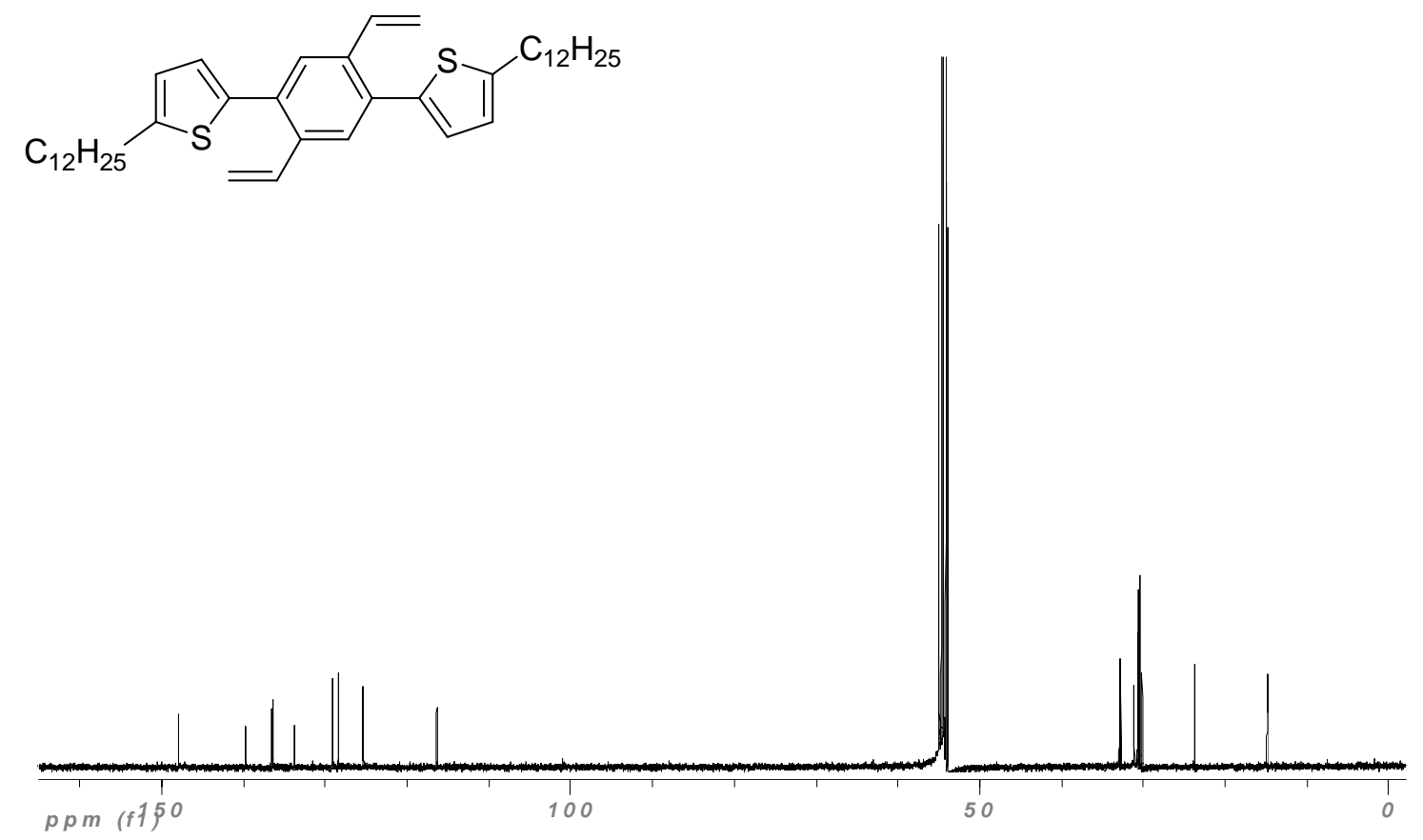

Figure 16. ${ }^{13} \mathrm{C}$ NMR spectrum of $\mathbf{4 d}\left(100 \mathrm{MHz}, \mathrm{CD}_{2} \mathrm{Cl}_{2}\right)$. 

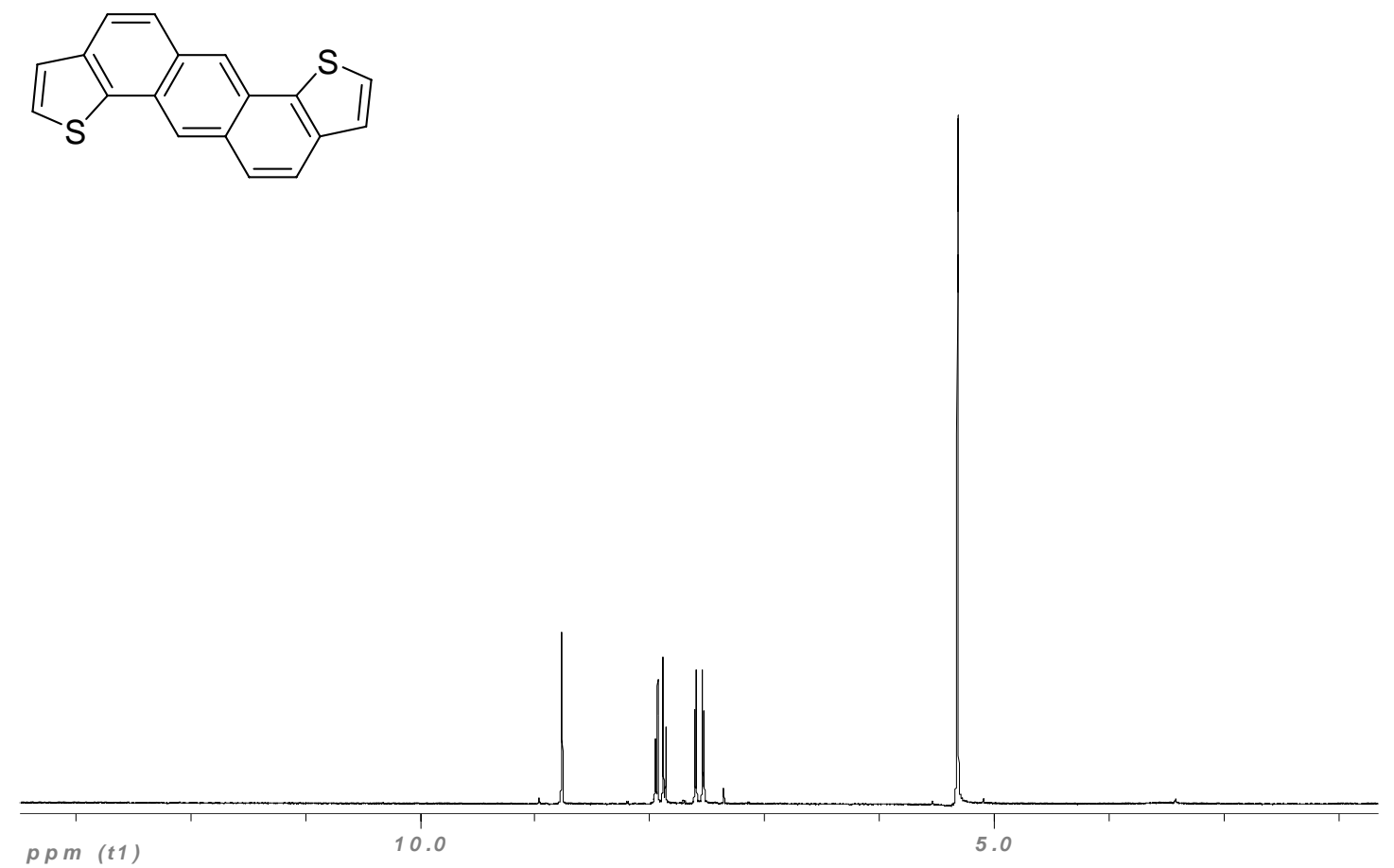

Figure 17. ${ }^{1} \mathrm{H} \mathrm{NMR}$ spectrum of $5 \mathbf{a}\left(400 \mathrm{MHz}, \mathrm{CD}_{2} \mathrm{Cl}_{2}\right)$.
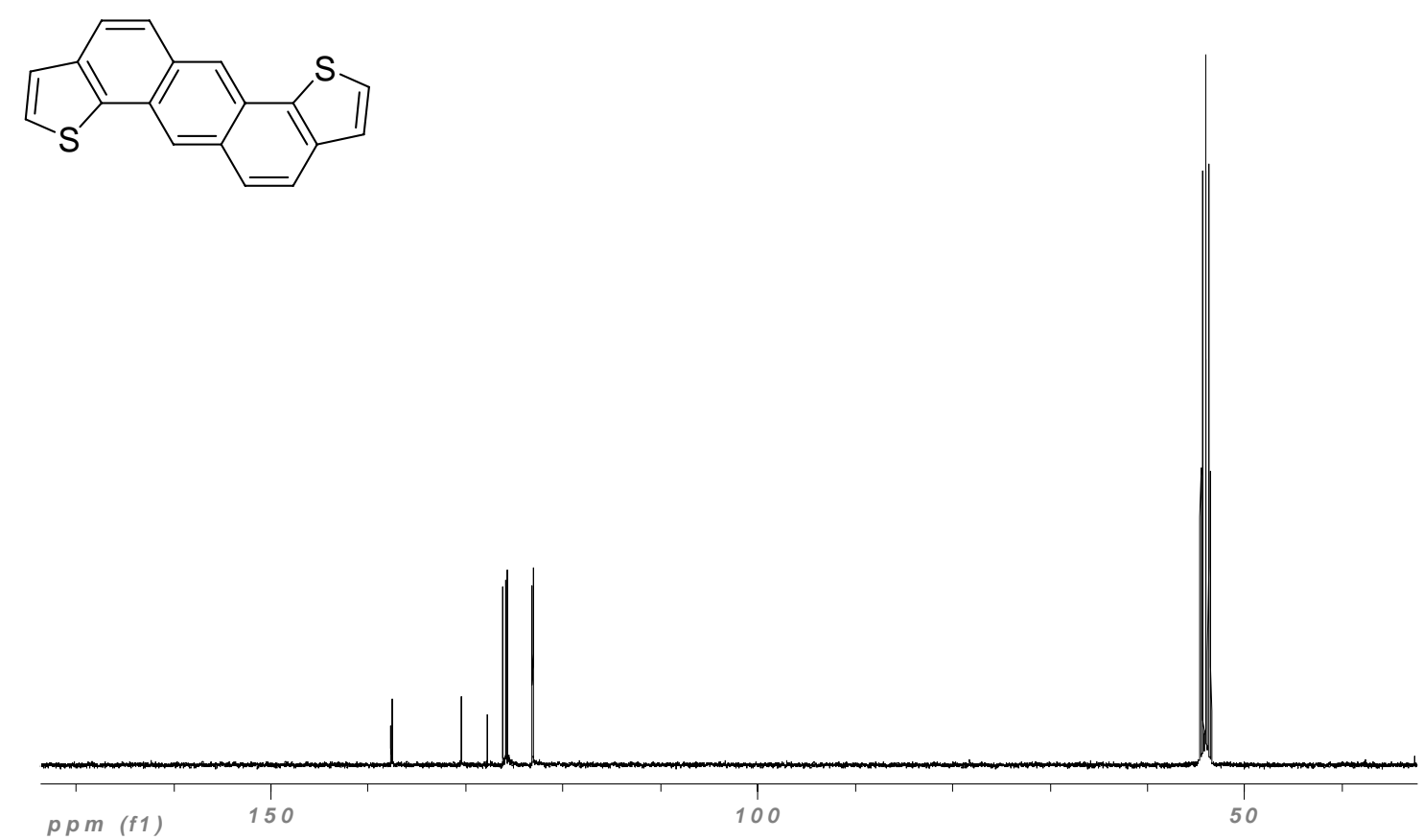

Figure 18. ${ }^{13} \mathrm{C}$ NMR spectrum of 5 a $\left(100 \mathrm{MHz}, \mathrm{CD}_{2} \mathrm{Cl}_{2}\right)$. 

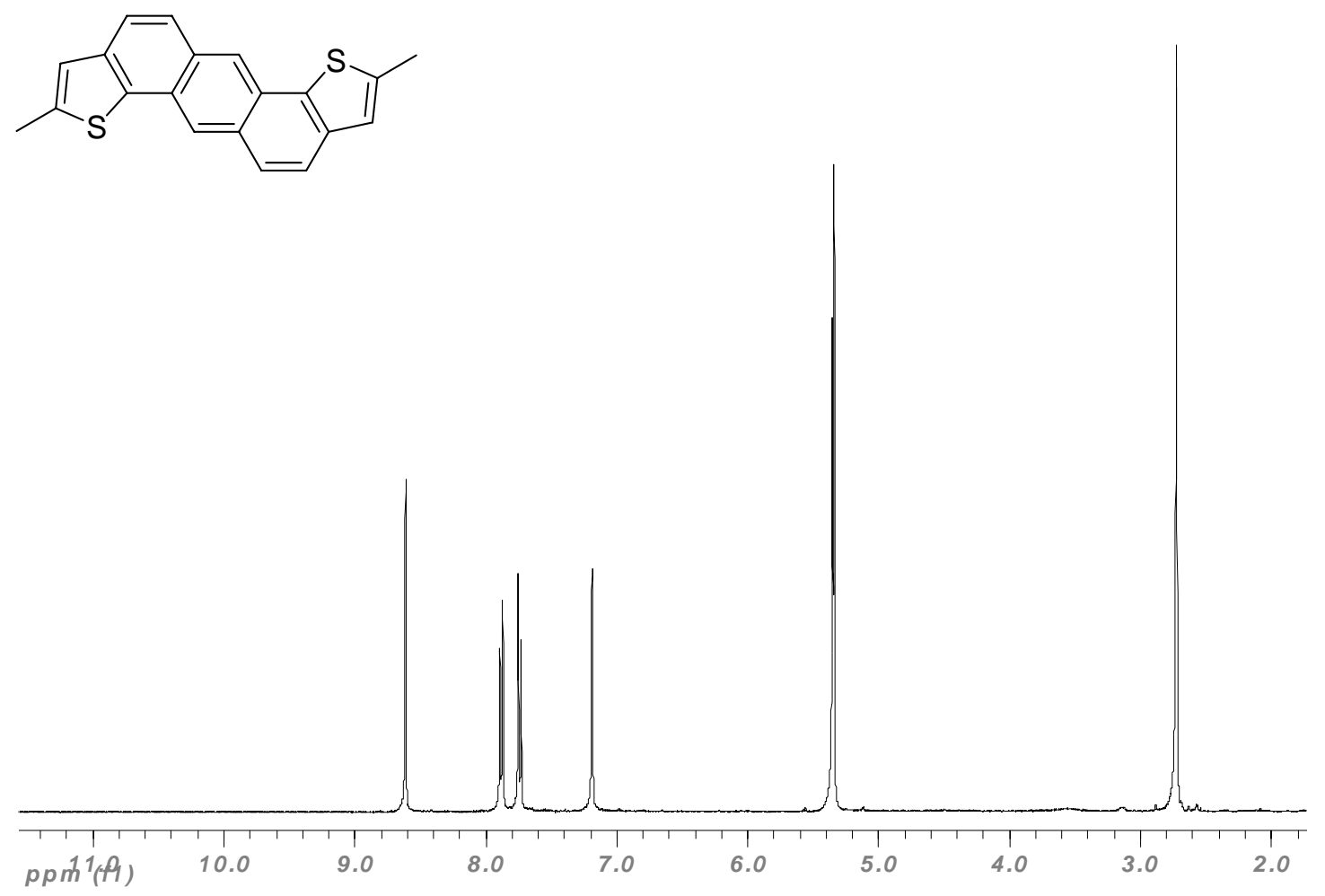

Figure 19. ${ }^{1} \mathrm{H} \mathrm{NMR}$ spectrum of $5 \mathbf{b}\left(400 \mathrm{MHz}, \mathrm{CD}_{2} \mathrm{Cl}_{2}\right)$.<smiles>Cc1cc2ccc3cc4c(ccc5cc(C)sc54)cc3c2s1</smiles>

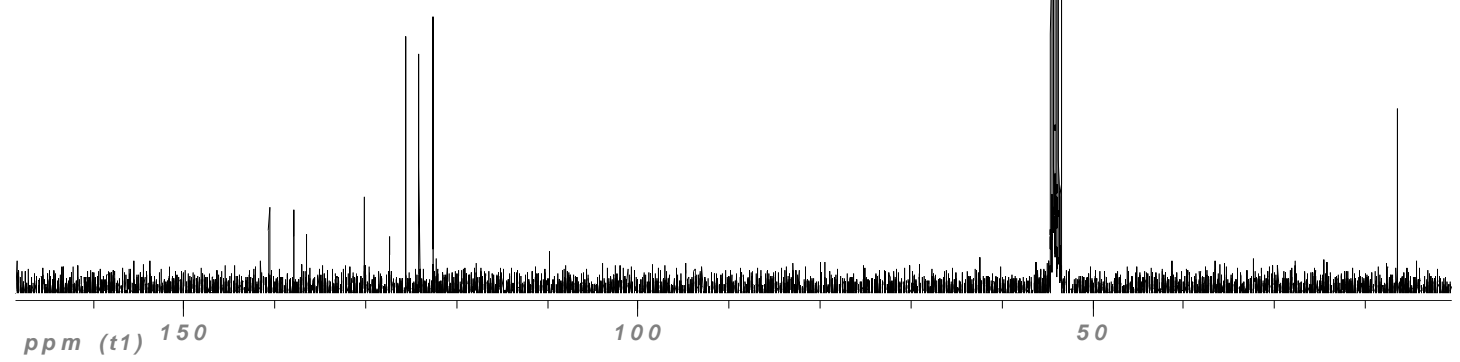

Figure 20. ${ }^{13} \mathrm{C}$ NMR spectrum of $5 \mathbf{b}\left(100 \mathrm{MHz}, \mathrm{CD}_{2} \mathrm{Cl}_{2}\right)$. 
<smiles>CCCCCCc1cc2ccc3cc4c(ccc5cc(CCCCCC)sc54)cc3c2s1</smiles>

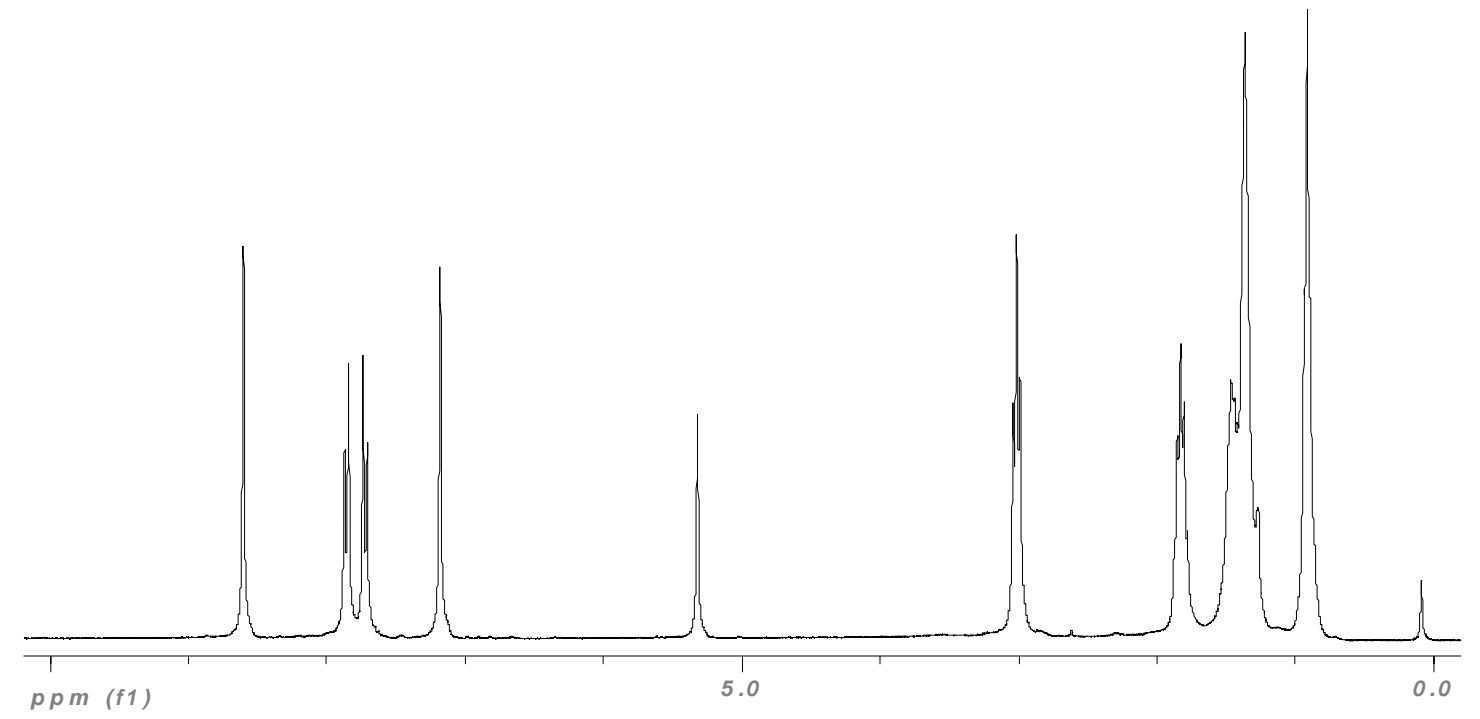

Figure 21. ${ }^{1} \mathrm{H}$ NMR spectrum of $5 c\left(400 \mathrm{MHz}, \mathrm{CD}_{2} \mathrm{Cl}_{2}\right)$.<smiles>CCCCCCc1cc2ccc3cc4c(ccc5cc(CCCCC)sc54)cc3c2s1</smiles>

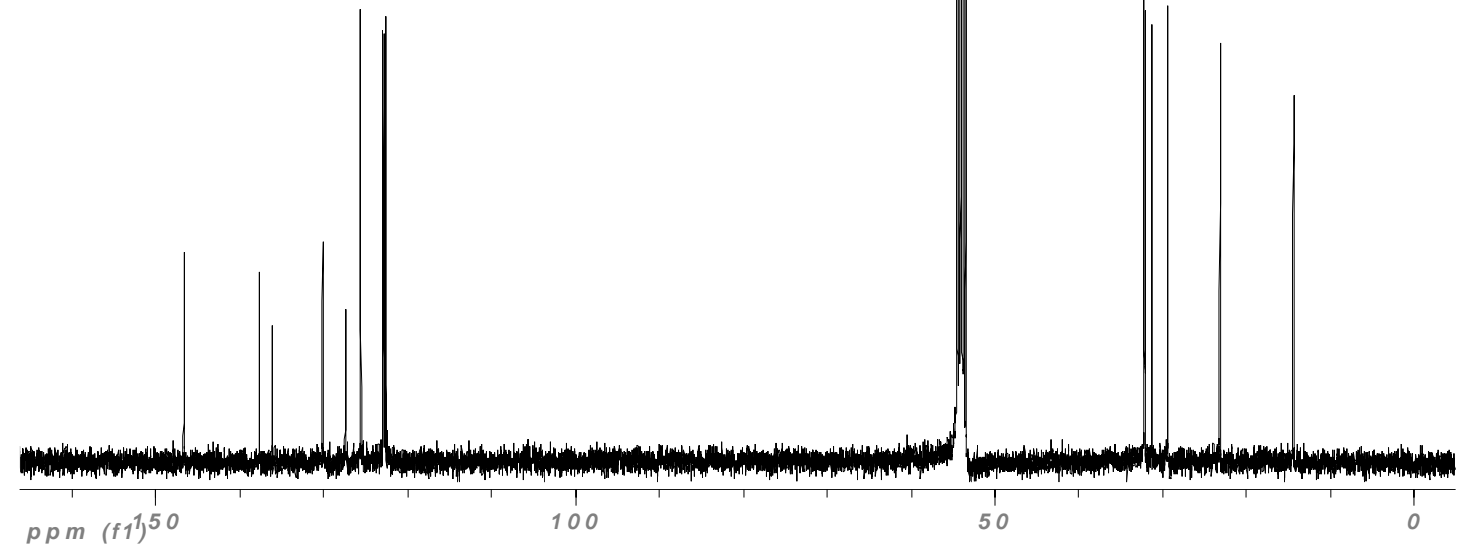

Figure 22. ${ }^{13} \mathrm{C}$ NMR spectrum of $5 c\left(100 \mathrm{MHz}, \mathrm{CD}_{2} \mathrm{Cl}_{2}\right)$. 


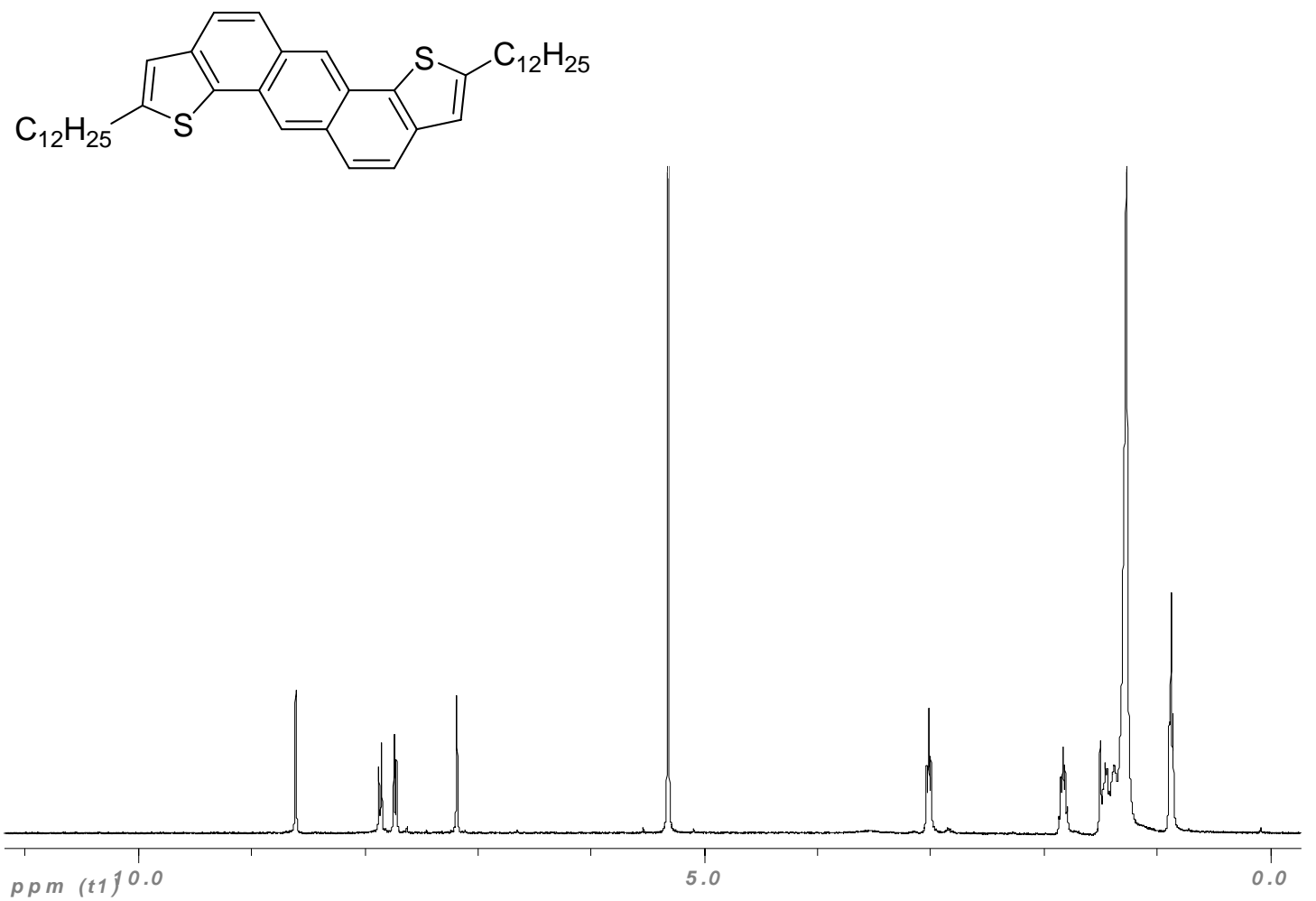

Figure 23. ${ }^{1} \mathrm{H} \mathrm{NMR}$ spectrum of $5 \mathbf{d}\left(400 \mathrm{MHz}, \mathrm{CD}_{2} \mathrm{Cl}_{2}\right)$.

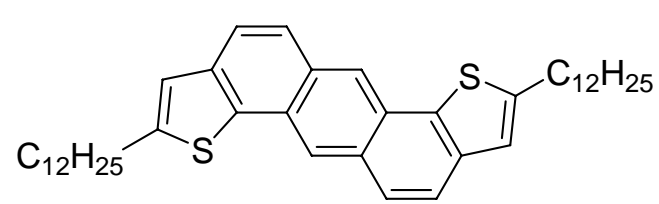

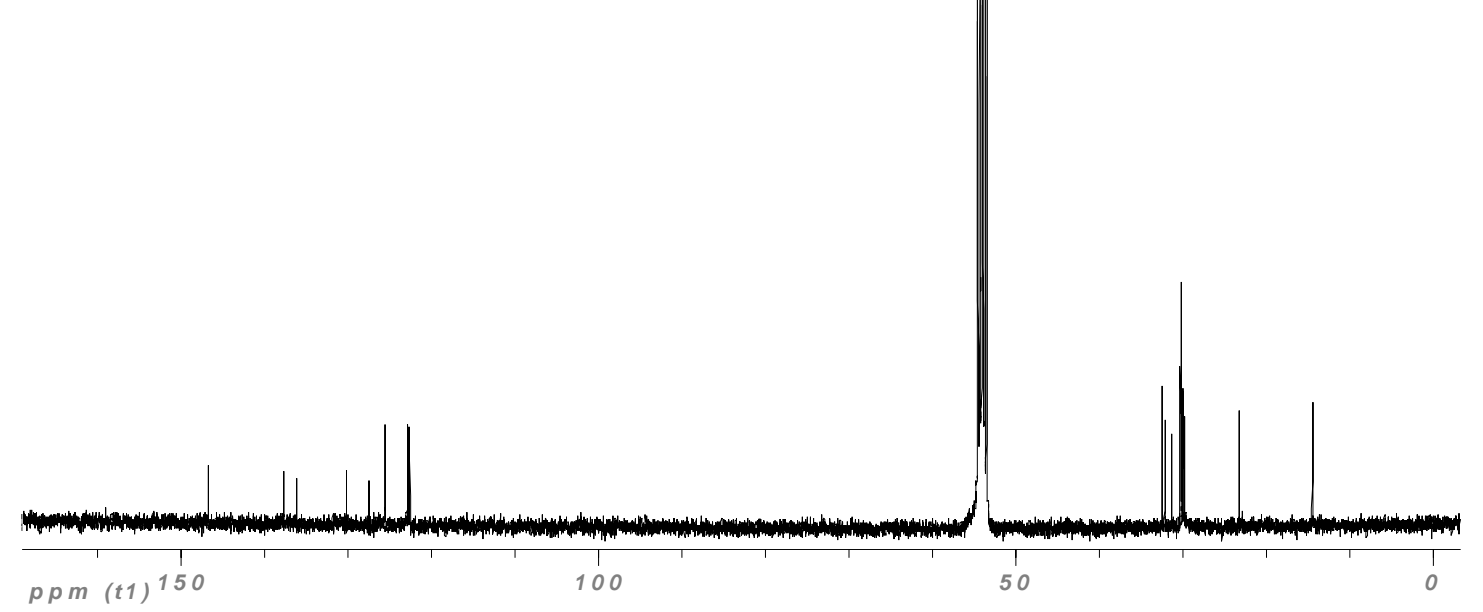

Figure 24. ${ }^{13} \mathrm{C}$ NMR spectrum of $5 d\left(300 \mathrm{MHz}, \mathrm{CD}_{2} \mathrm{Cl}_{2}\right)$. 


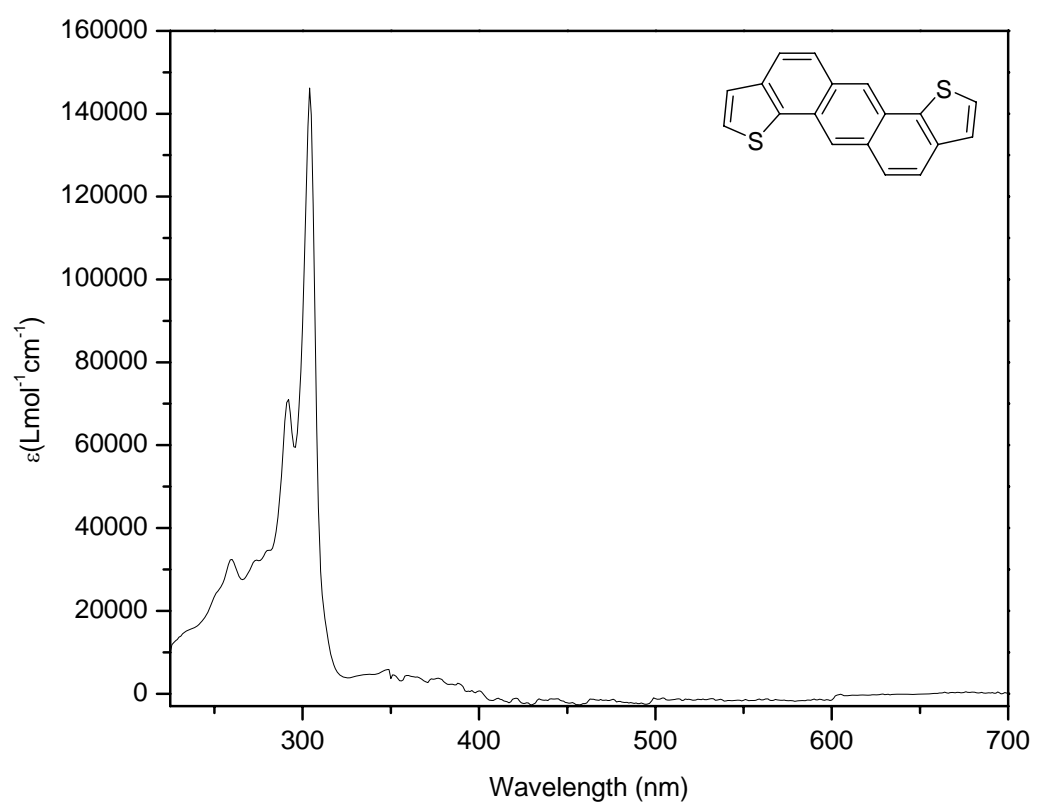

Figure 25. UV/vis absorption spectrum of 5 a.

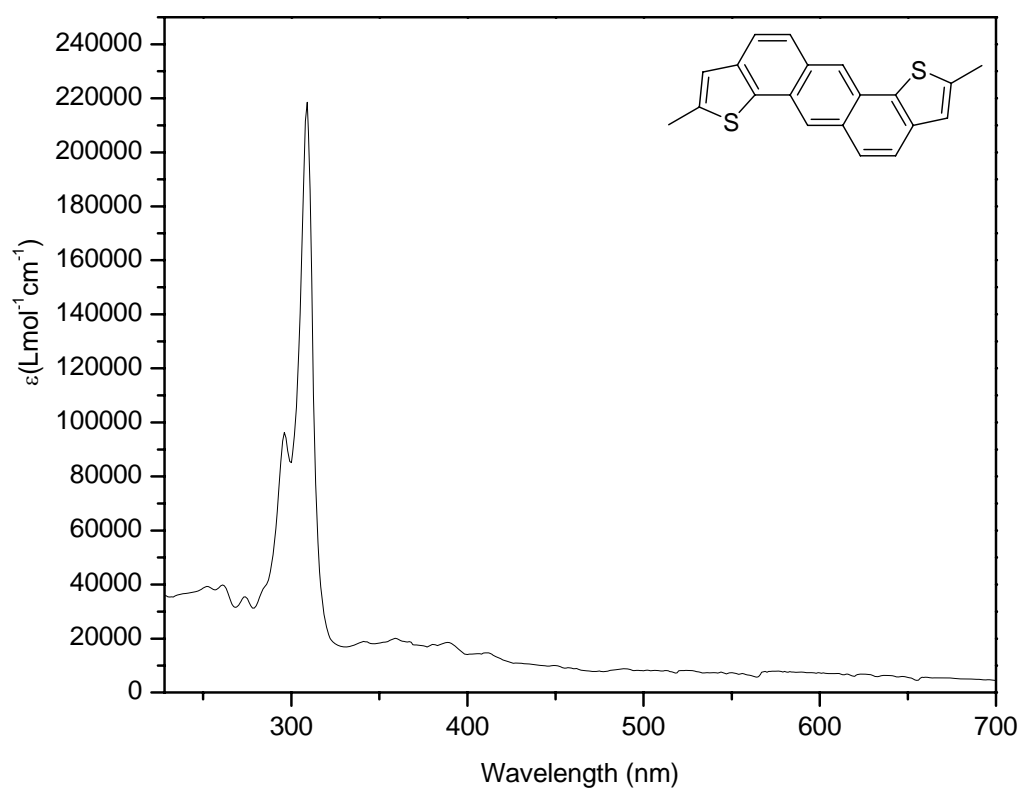

Figure 26. UV/vis absorption spectrum of $5 \mathbf{b}$. 


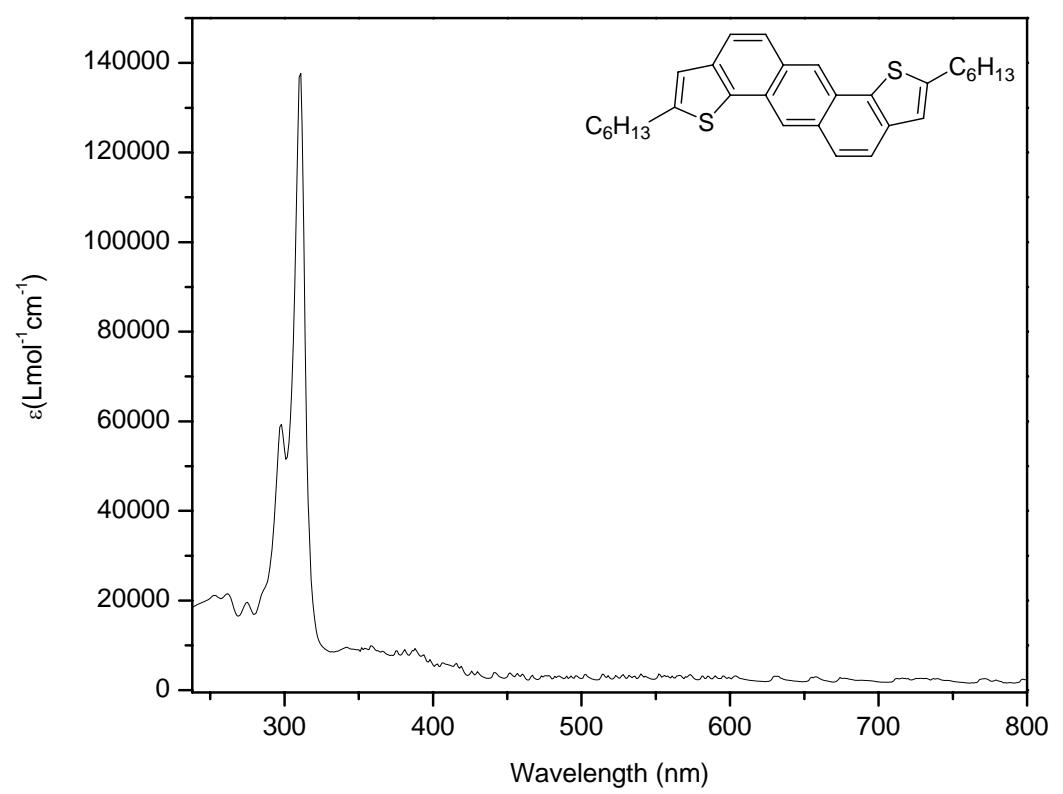

Figure 27. UV/vis absorption spectrum of 5c.

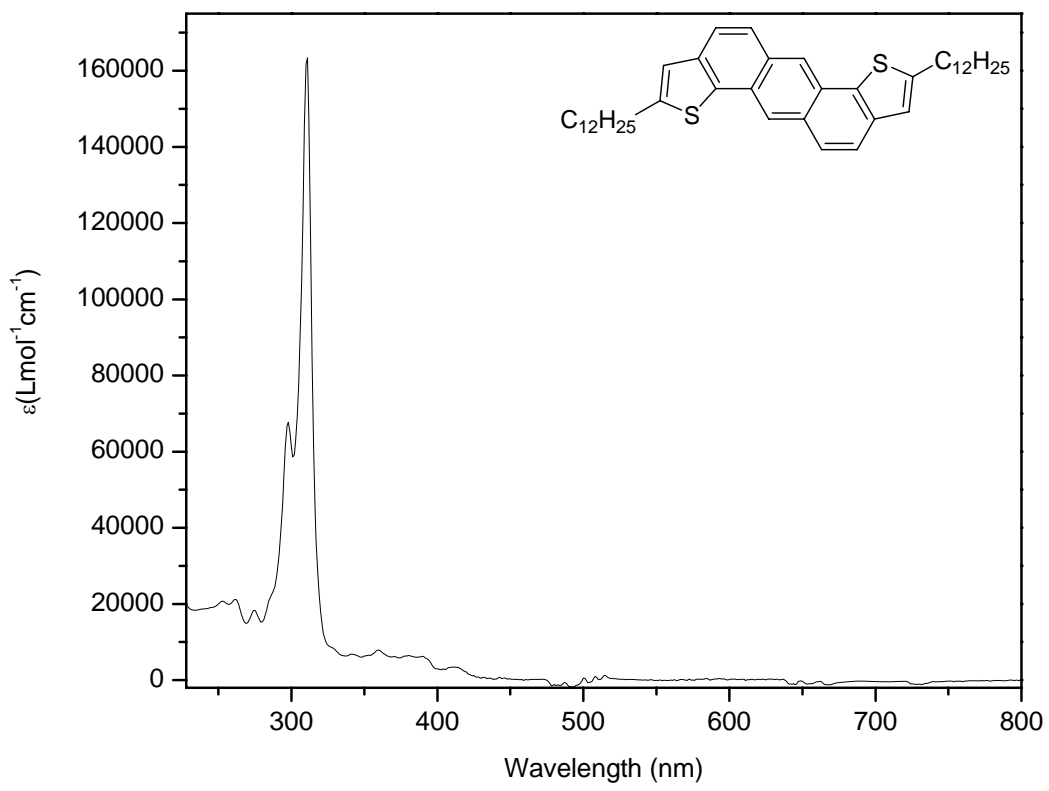

Figure 28. UV/vis absorption spectrum of $\mathbf{5 d}$. 


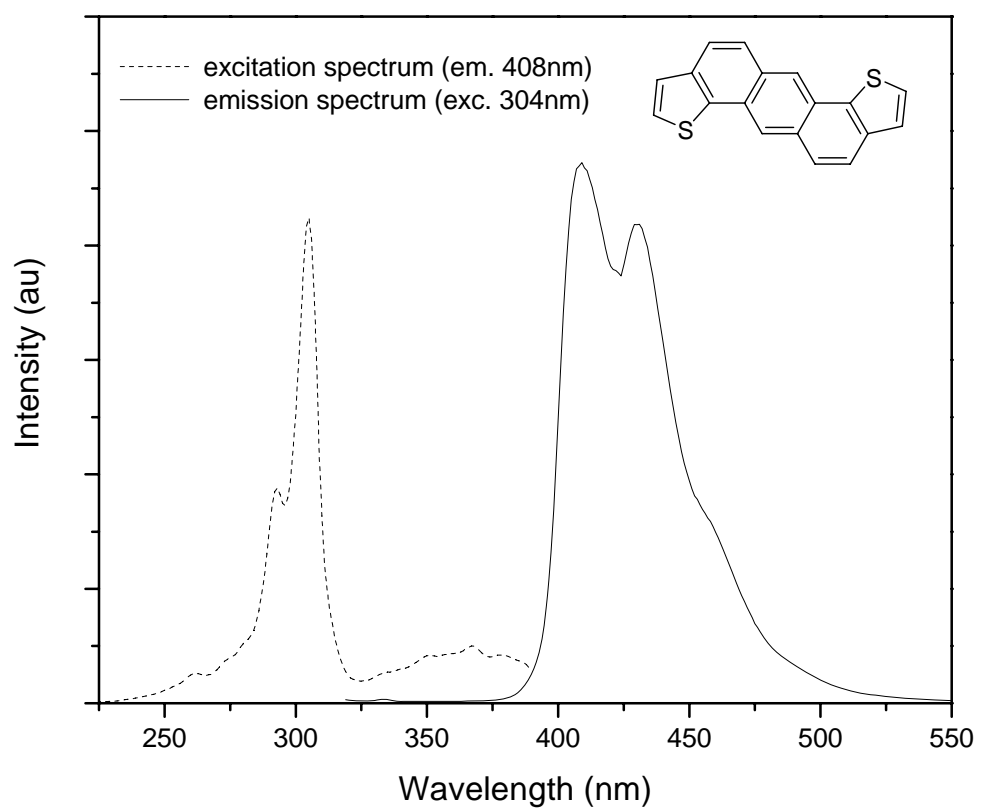

Figure 29. Excitation and emission spectra of 5 a.

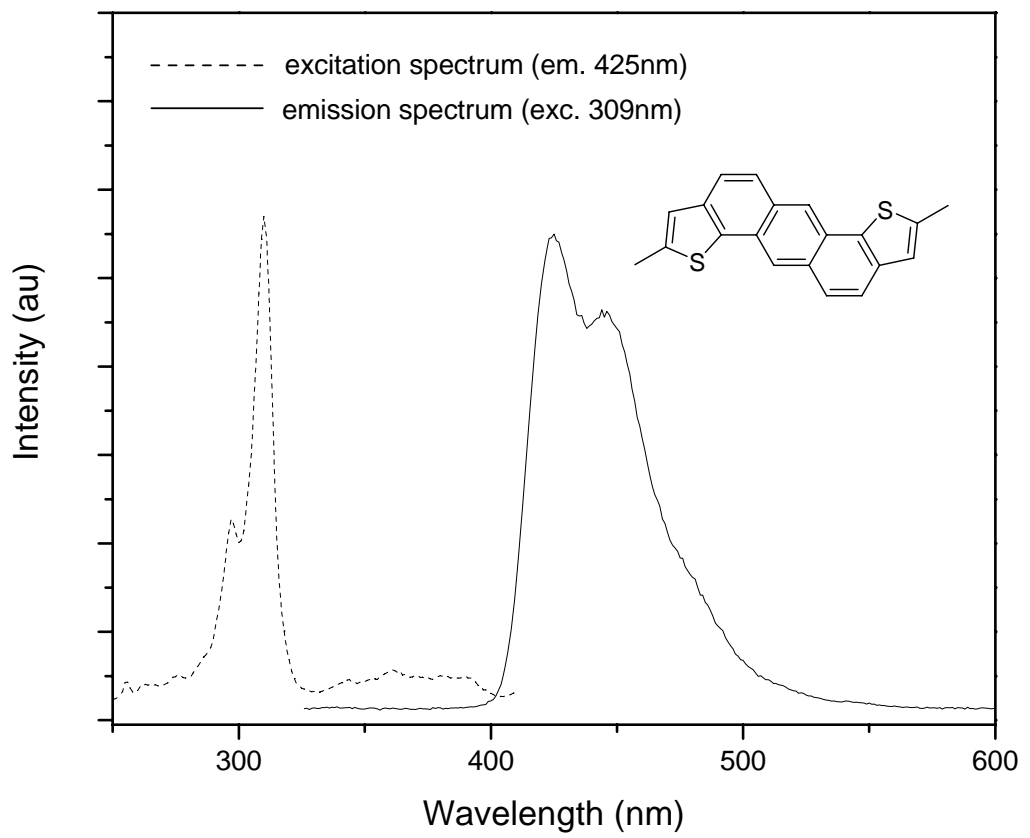

Figure 30. Excitation and emission spectra of $5 \mathbf{b}$. 


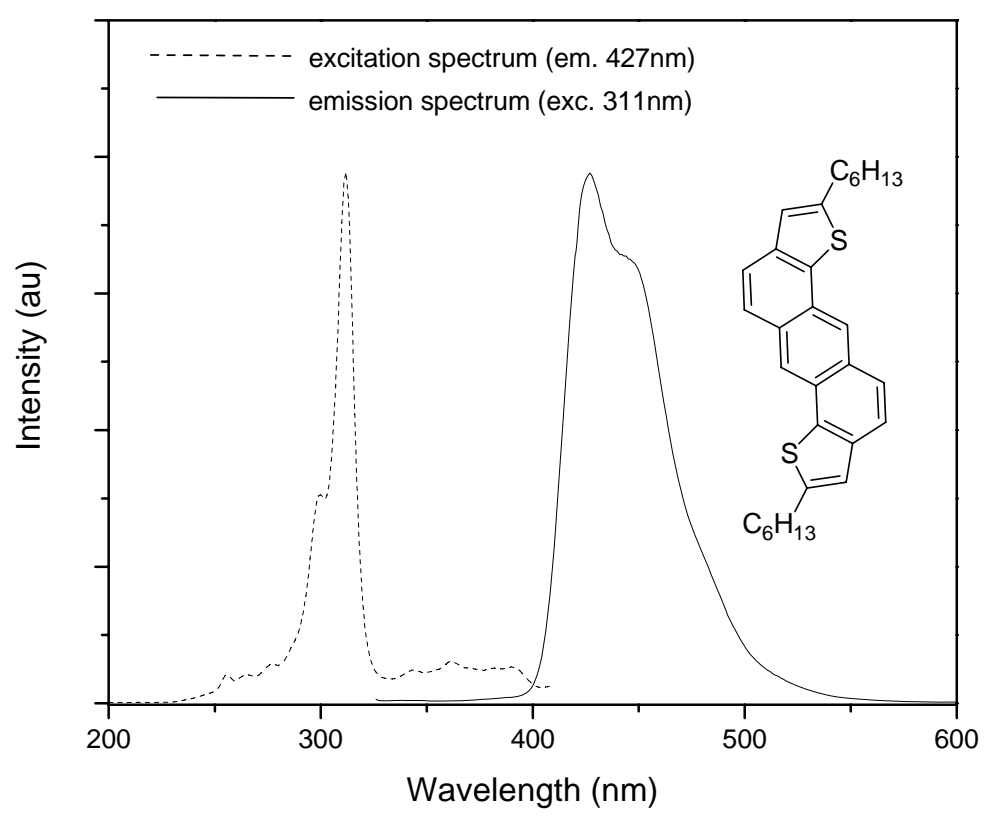

Figure 31. Excitation and emission spectra of 5c.

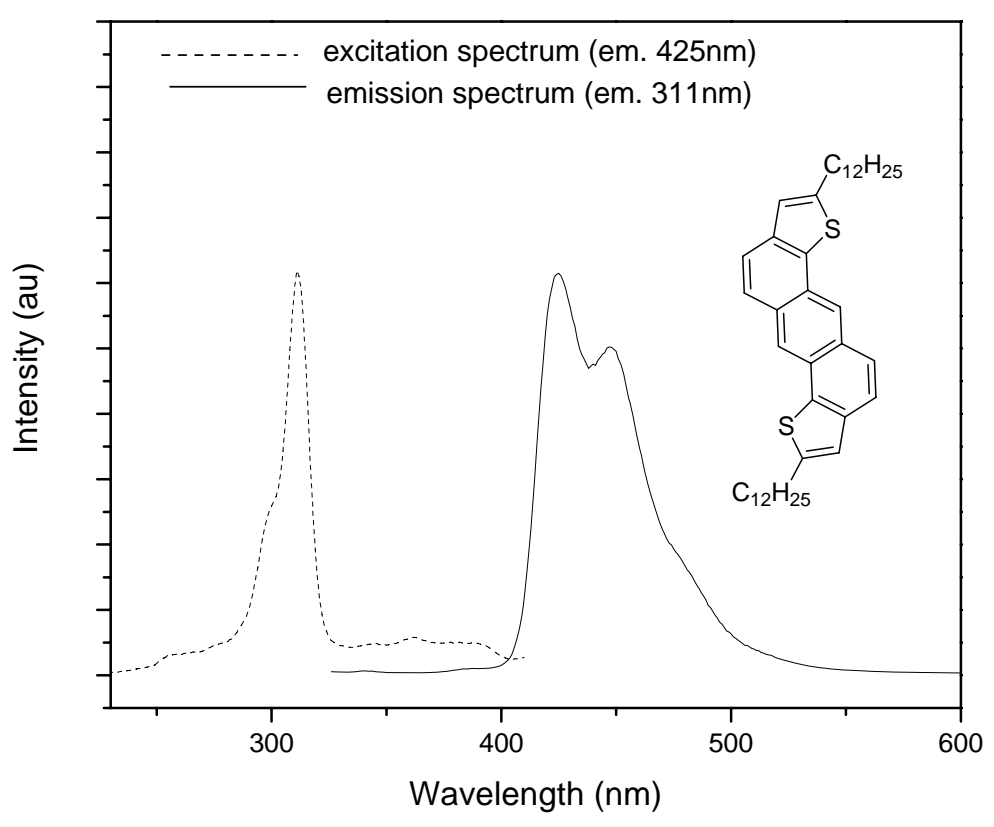

Figure 32. Excitation and emission spectra of 5d. 
${ }^{1}$ Chen, Z.-K.; Huang, W.; Wang, L.-H.; Kang E.-T., Chen, B.J.; Lee, C. S.; Lee, S. T. Macromolecules 2000, 33, 9015.

${ }^{2}$ Xia, C.; Advincula, R. C. Macromolecules 2001, 34, 6992.

${ }^{3}$ Berridge, R.; Wright, S. P.; Skabara, P. J.; Dyer, A.; Steckler, T.; Argun, A. A.;

Reynolds, J. R.; Harrington, R. W.; Clegg, W. J. Mater. Chem. 2007, 17, 225.

${ }^{4}$ Jousselme, B.; Blanchard, P.; Gallego-planas, N.; Levillain, E.; Delaunay, J.; Allain, M.; Richomme, P.; Roncali, J. Chem. Eur. J. 2003, 9, 5297.

${ }^{5}$ Wilson, P.; Lacey, D.; Sharma, S.; Worthington, B. Mol. Cryst. Liq. Cryst. Sci. Tech. Sect. A: Mol. Cryst. Liq. Cryst. 2001, 368, 279.

${ }^{6}$ Weber, G.; Teale, F. W. J. Trans. Faraday Soc. 1957, 53, 646. 